\title{
Is Cancer Recurrence Postponed By a Treatment? A New Model Answers
}

\author{
Ramalingam Shanmugam \\ School of Health Administration, Texas State University, TX 78666 San Marcos, USA
}

Received 2012-08-12, Revised 2012-12-24; Accepted 2013-03-26

\begin{abstract}
During a treatment period of a cancer patient, the medical team ponders over a question: Is patient's cancer recurrence significantly postponed by the medication, even if the illness is not completely cured? This question could be answered with data evidence if the data are analyzed with an appropriate model and a suitable methodology. A scanning of the literature reveals that no suitable technique now exists. Hence, there is a need to develop a new probability model and to formulate a statistical methodology. Both tasks are accomplished in this article to answer the above question. The Exponential Distribution (ED) is traditionally employed to portray the chance for a patient to survive beyond a given time, $t>0$. Because of its memory less property, the exponential distribution lacks an ability to answer whether the medication has significantly postponed the cancer recurrence for a patient. In essence, this memory less property assumes that a patient's body functions with no memory. This notion of memory less is quite unreal with respect to cancer recurrence as it is contradictory to the medical belief of medicating a patient with an intention to stop or at least postpone cancer recurrence in a disease management. This article takes an approach by adding a prolonging parameter, $\rho \geq 0$ to the exponential distribution to represent the medication's effect on the survival chance for a patient without a cancer recurrence. Because of the prolonging parameter, the probability structure of the exponential distribution gets altered and it is explicitly explained in the article. The altered exponential distribution is named Prolonged Survival Exponential Distribution (PSED). The properties of PSED are derived and then utilized to analyze patients' cancer recurrence data. Also, likelihood ratio based statistical methodology based on PSED is derived and illustrated to address whether the medication has significantly postponed the cancer's recurrence time in a particular patient. An analysis of recurrence times of three groups of sixteen bladder cancer patients was done. The first is the placebo group with seven patients. The second and third group consist respectively three and six patients receiving thiotepa and pyridoxine medication. The probability plots of their recurrence times confirm that the data do not follow an exponential distribution. The data analyses results reveal interesting statistical information about how effective the medication had been on each individual patient. Without the new model, PSED and the likelihood ratio testing methodology of this article, a patient specific interpretation is not possible. The medication worked in some but not all patients. The medication is thought to have not worked in a patient, when his/her estimate, $\rho$ is closer to zero. However, on the average, the 2 nd bladder cancer recurrence time is quicker in place group, next in pyridoxine group and much later in tholepin group. The new model, PSED and the likelihood ratio test of this article would be useful to analyze and interpret the probability pattern of recurrence times and whether the medication has significantly prolonged the next recurrence time of other cancers like melanoma, ulcer, ovarian. Also, the contents of this article have established a foundation to build a generalized regression methodology to identify predictors for increasing the survival time of cancer patients with more patients' data.
\end{abstract}

Keywords: Likelihood Ratio, Exponential Distribution, Power, P-Value, Memory Less Property, Survival Function 


\section{INTRODUCTION}

Does cancerincidence data reveal that a medication to the patient has prolonged at least the recurrence time, even if the medication has not completely cured the illness? To be specific, let $\mathrm{Y}$ be a cancer recurrence time. How should one configure $Y$ to confirm the medication's effectiveness? No suitable technique now exists in the literature to answer this question. A data analytic approach is necessary. A development of such a comprehensive approach is the aim of this article and it requires selection of an appropriate model for Y. Could exponential distribution (Stuart and Ord, 1994) with the survival function:

$\mathrm{S}_{\text {exp }}[\mathrm{y} \mid \tau]=\operatorname{Pr}[\mathrm{Y}>\mathrm{y} \mid \tau]=\mathrm{e}^{-\mathrm{y} / \tau}, \tau>0, \mathrm{y} \geq 0$

plays a role for $Y$, with $\tau>0$ denoting the expected time for next recurrence? Panjer (2006) for details on how model in (1) and others are used to capture uncertainty. The survival function, $S_{\exp }[y \mid \tau]$ The model (1) lacks ability to predict the next cancer recurrence. Why is it so? Reasonsare explored and stated below in details.

The conditional survival probability for a patient to experience arecurrence in the next $\mathrm{m}$ months given that $\mathrm{s} / \mathrm{h}$ did not experience a cancer in $\mathrm{t}$ months is same the unconditional survival probability of not experiencing one in any time period (including in the beginning) of same length of $\mathrm{m}$ months. Rephrasing it in probability terms, it means:

$$
\begin{aligned}
& \operatorname{Pr}[\mathrm{Y}>\mathrm{t}+\mathrm{m} \mid \mathrm{Y}>\mathrm{t}] \\
& =\frac{\operatorname{Pr}[\mathrm{Y}>\mathrm{t}+\mathrm{m}]}{\operatorname{Pr}[\mathrm{Y}>\mathrm{t}]} \\
& =\mathrm{e}^{-\mathrm{m} / \tau} \\
& =\operatorname{Pr}[\mathrm{Y}>\mathrm{m}]
\end{aligned}
$$

This feature in Equation (2) is referred memory-less property. Would a cancer cell function with no memory, especially when a patient receives a medication? If the answer is "yes", the medication is obviously meaningless. None in medical community might agree with no-memory operation style of a cancerous cell. The answer ought to be "no" intuitively, since it is real and medically meaningful. Furthermore, the survival probability in Equation (1) diminishes as time, $t$ progresses. Shouldn't a medication elevate the survival probability? The exponential distributionis, therefore, not in harmony with medical expectations as it does not have a feature to explain the role of medication's effect on the survival chance. Hence, the model (1) needs an alteration. Also, the exponential distribution indicates a constant expected recurrence time:

$$
E[Y]=\int_{0}^{\infty} y d\left(1-S_{e x p}[y \mid \tau]\right)=\tau
$$

Is the above stated constant expected cancer recurrence time realistic? Wouldn't a medication prolong the expected recurrence time, at the least? The exponential distribution is not realistic enough to illustrate the prolonging aspect of next cancer recurrence due to medication's effect. A new model to portray the prolonging of recurrence time due to the medication's effect is necessary and it is derived in this article by altering the exponential distribution.

Before proceeding further to make alteration, why not look at real data sets in Table 1 of bladder cancer recurrence times to check whether the data support for a need to alter? The data sets are from Lindsey (1997) the entry in Table $\mathbf{1}$ is $\mathrm{Y}$, the number of months waited to experience a recurrence in three groups of $n_{1}=7 n_{2}=3$ and $n_{3}=6$ bladder cancer patients (Fig. 1). The groups received respectively: placebo, thiotepa and pyridoxine. The placebo commonly refers to ineffectual medication's effect to deceive the recipient. Beecher (1955) argues that placebo does not work in every clinical trial. The drug "Tiotepa" was first developed by the American Cyanamid Company in year 1950 and it is an alkylating substance given to cancer patients with or without body irradiation. See www.cancer.org for clinical details about thiotepa drug. The drug "Pyridoxine" (an extract from grains, nuts and dragon fruit) helps the body to balance sodium and potassium to increase red blood cell production and stabilize hormonal changes. Kashanian et al. (2007) for details on medicinal use of pyridoxine. The exponential probability plots of the data sets in Table 1 are displayed in Fig. 2a through $2 \mathrm{~h}$ below. For the data to have come from an exponential distribution, the data points should be closer to the diagonal line. Did that happen? Clearly, that is not the case. Hence, it is reasonable to infer that the bladder cancer recurrence times of the sixteen patients have not come from an exponential distribution. The clue exists in the data sets that the medication might have worked in the patients. Consequently, their survival chance without a recurrence might have been elevated because of the medication's effect.

An altered probability model is introduced before with its properties. The new model is named Prolonged Survival Exponential Distribution (PSED). The Maximum Likelihood Estimators (MLE) of PSED and a likelihood ratio based hypothesis testing procedure are developed to assess the significance of the medication's effect to prolong the recurrence time in Fig. 3. (a) through (d). 
Ramalingam Shanmugam / American Medical Journal 4 (1): 43-62, 2013

Table 1. Bladder cancer recurrence times in months for placebo, thiotepa and pyridoxine groups

\begin{tabular}{llrrrrrrrrrrrr}
\hline Patient & Group & $1 \mathrm{st}$ & 2nd & 3rd & 4th & 5 th & 6 th & 7 th & 8th & $\bar{y}$ & $\mathrm{~s}_{\mathrm{y}}^{2}$ & $\bar{\tau}$ & $\bar{\rho}$ \\
\hline 1 & placebo & 3 & 3 & 2 & 4 & 14 & & & & 5 & 25.0 & 4.75 & 0.02 \\
2 & placebo & 16 & 3 & 4 & 6 & 5 & 6 & & & 7 & 22.0 & 3.34 & 74.70 \\
3 & placebo & 9 & 2 & 9 & 6 & 4 & & & & 6 & 9.5 & 1.58 & 0.98 \\
4 & placebo & 3 & 12 & 31 & 5 & 2 & & & & 11 & 145.0 & 13.70 & 0.01 \\
5 & placebo & 2 & 13 & 9 & 6 & 4 & 5 & 4 & 6 & 6 & 11.0 & 1.98 & 1.06 \\
6 & placebo & 5 & 9 & 5 & 8 & 14 & & & & 8 & 14.0 & 1.67 & 0.80 \\
7 & placebo & 2 & 6 & 4 & 1 & 4 & 4 & 12 & 16 & 6 & 27.0 & 4.41 & 0.15 \\
1 & thiotepa & 1 & 2 & 2 & 2 & 3 & & & & 2 & 0.5 & 0.25 & 4.67 \\
2 & thiotepa & 4 & 12 & 7 & 4 & 6 & 3 & 1 & & 5 & 13.0 & 2.38 & 2.31 \\
3 & thiotepa & 2 & 18 & 3 & 4 & 11 & & & & 8 & 46.0 & 6.09 & 0.05 \\
1 & pyridoxine & 3 & 7 & 12 & 4 & 8 & & & & 7 & 13.0 & 1.87 & 0.86 \\
2 & pyridoxine & 3 & 6 & 6 & 4 & 6 & & & & 5 & 2.0 & 0.40 & 2.74 \\
3 & pyridoxine & 3 & 4 & 5 & 4 & 3 & 9 & 6 & 2 & 4 & 4.5 & 1.04 & 1.41 \\
4 & pyridoxine & 2 & 4 & 4 & 6 & 7 & 4 & 9 & 3 & 5 & 5.0 & 1.07 & 1.33 \\
5 & pyridoxine & 8 & 7 & 3 & 2 & 2 & 3 & 13 & 2 & 5 & 16.0 & 3.20 & 0.40 \\
6 & pyridoxine & 8 & 6 & 6 & 5 & 4 & 4 & 15 & 1 & 6 & 17.0 & 2.77 & 2.09 \\
\hline
\end{tabular}

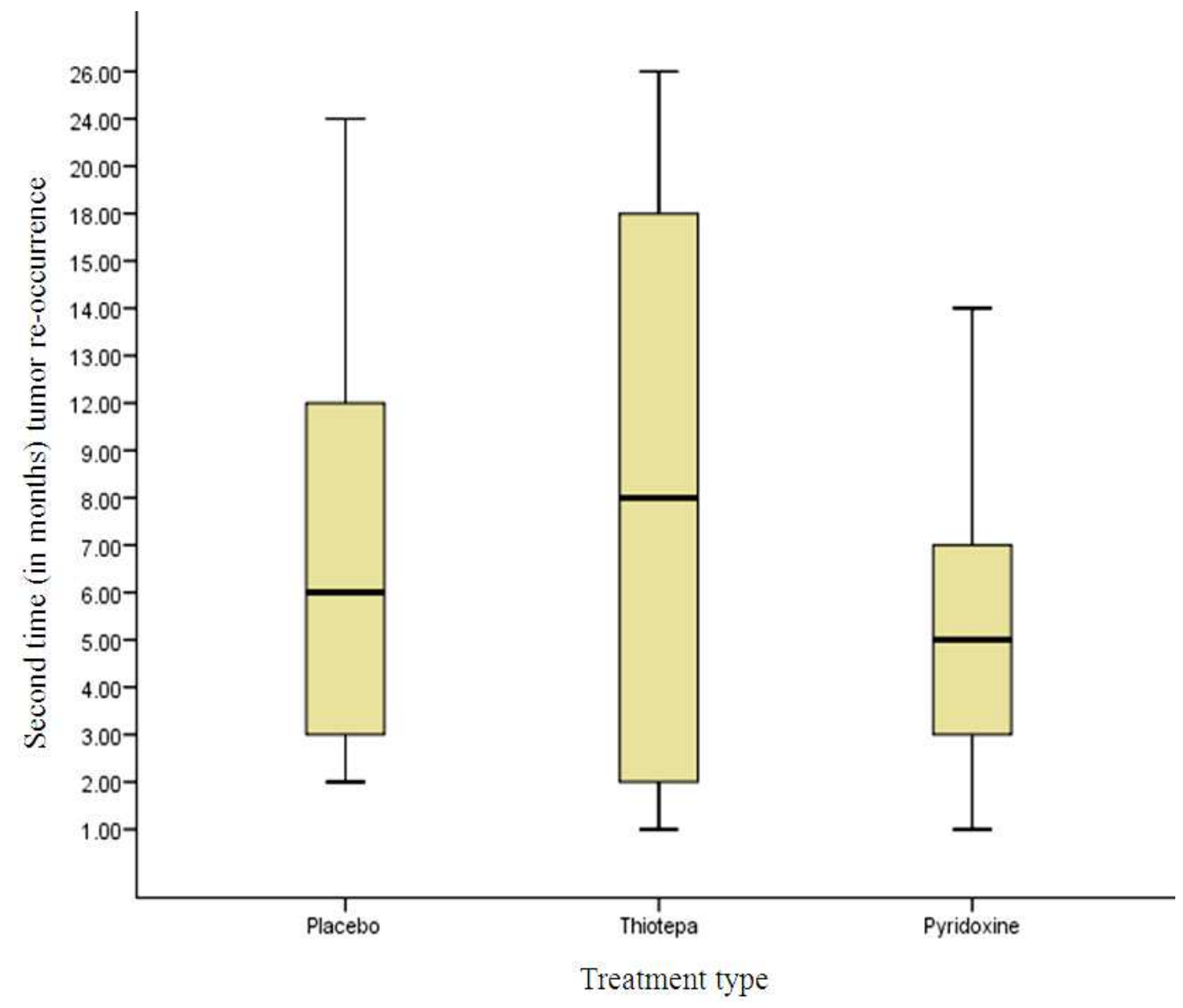

Fig. 1. Box plot of bladder cancer recurrence times in three medication's effect groups 
Ramalingam Shanmugam / American Medical Journal 4 (1): 43-62, 2013

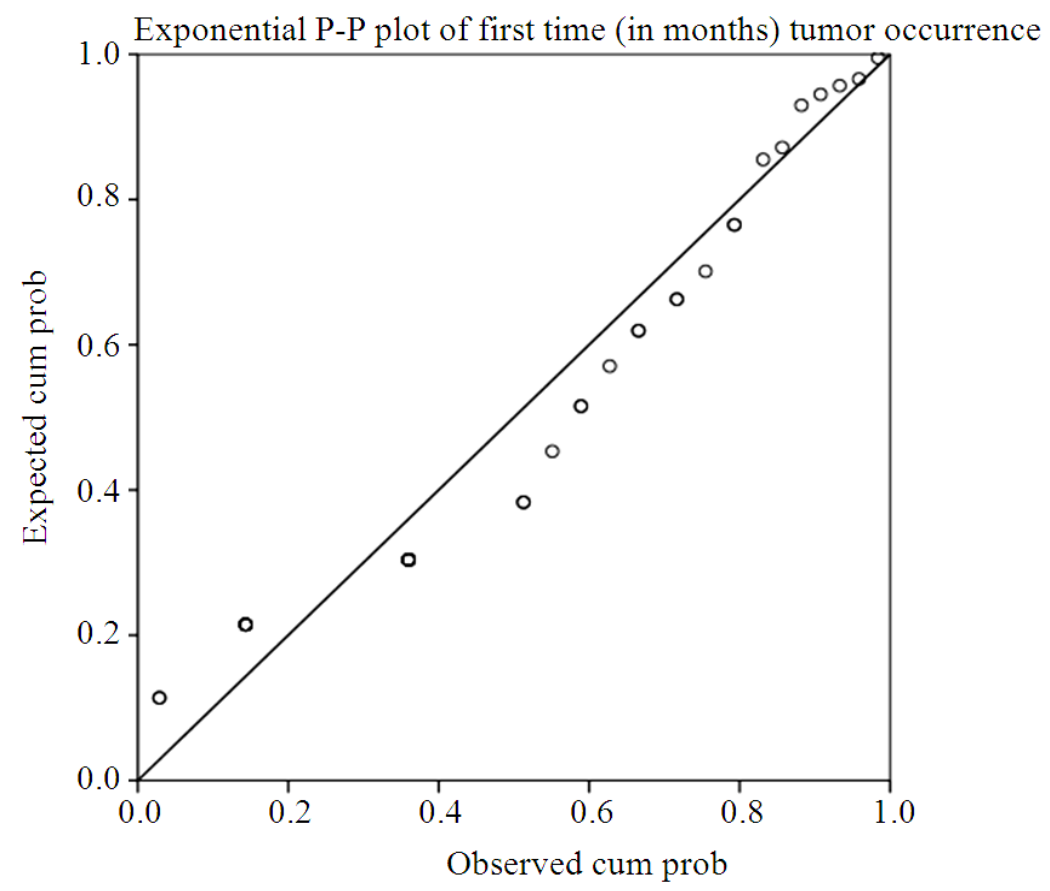

(a)

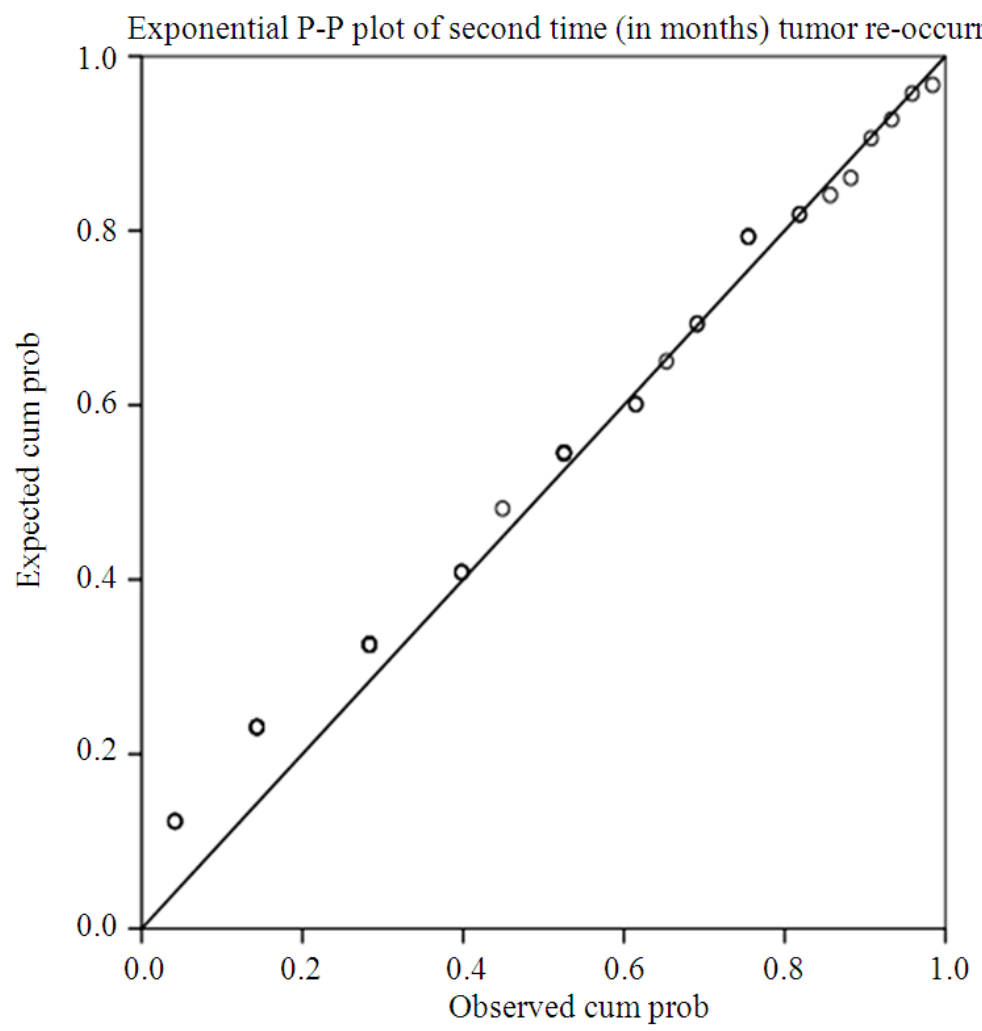

(b) 
Ramalingam Shanmugam / American Medical Journal 4 (1): 43-62, 2013

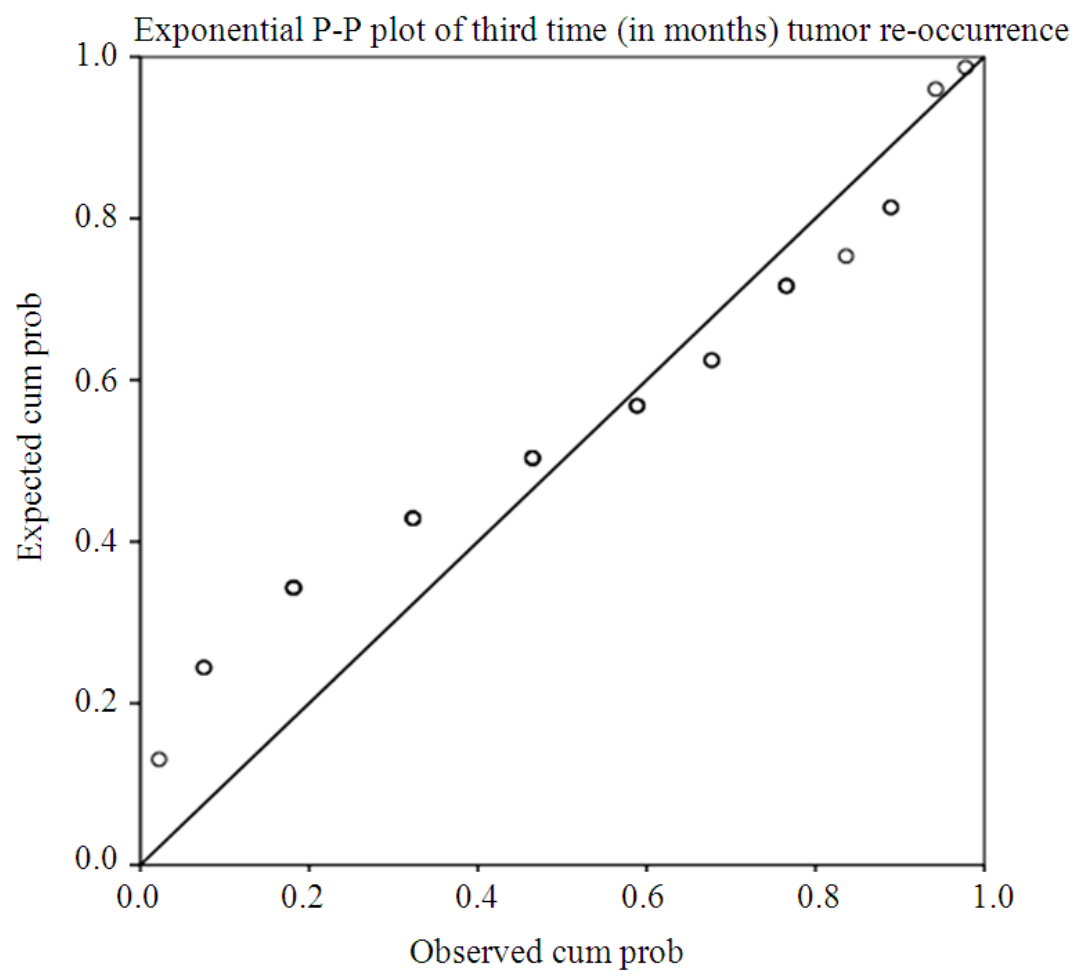

(c)

Exponential P-P plot of fourth time (in months) tumor re-occurrence

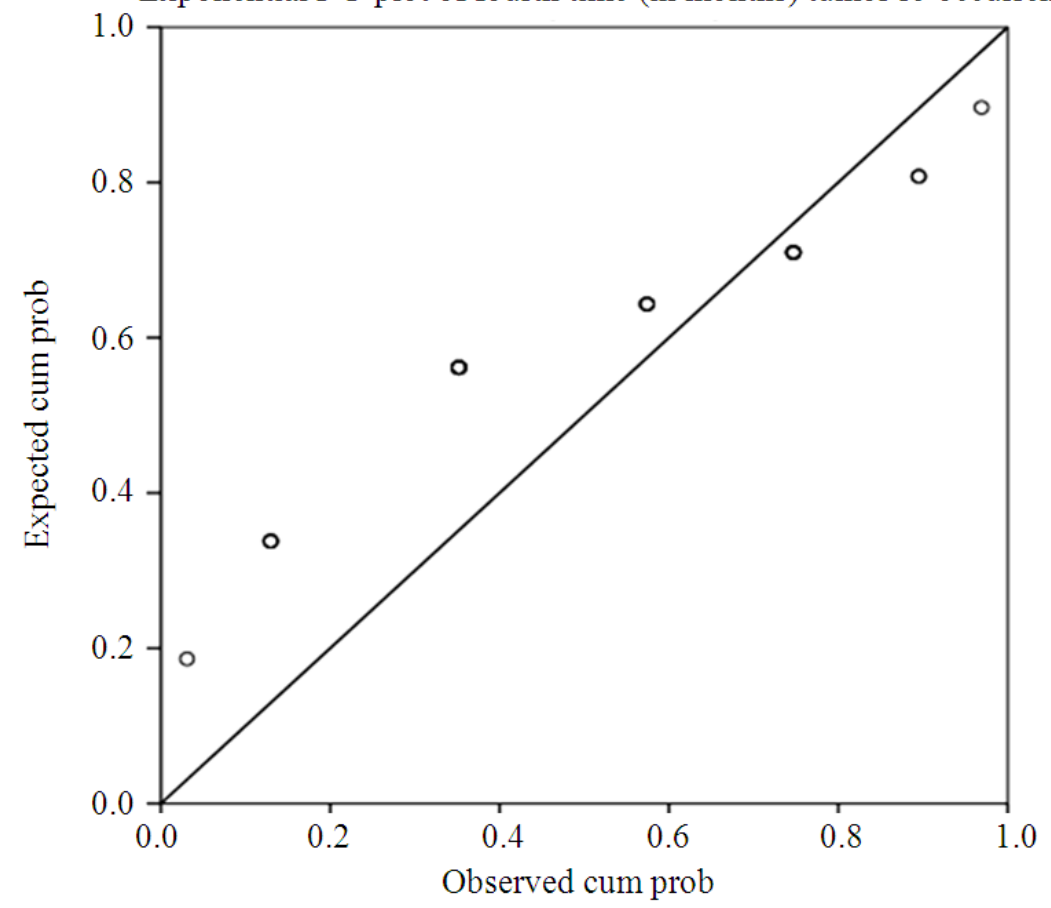

(d) 
Ramalingam Shanmugam / American Medical Journal 4 (1): 43-62, 2013

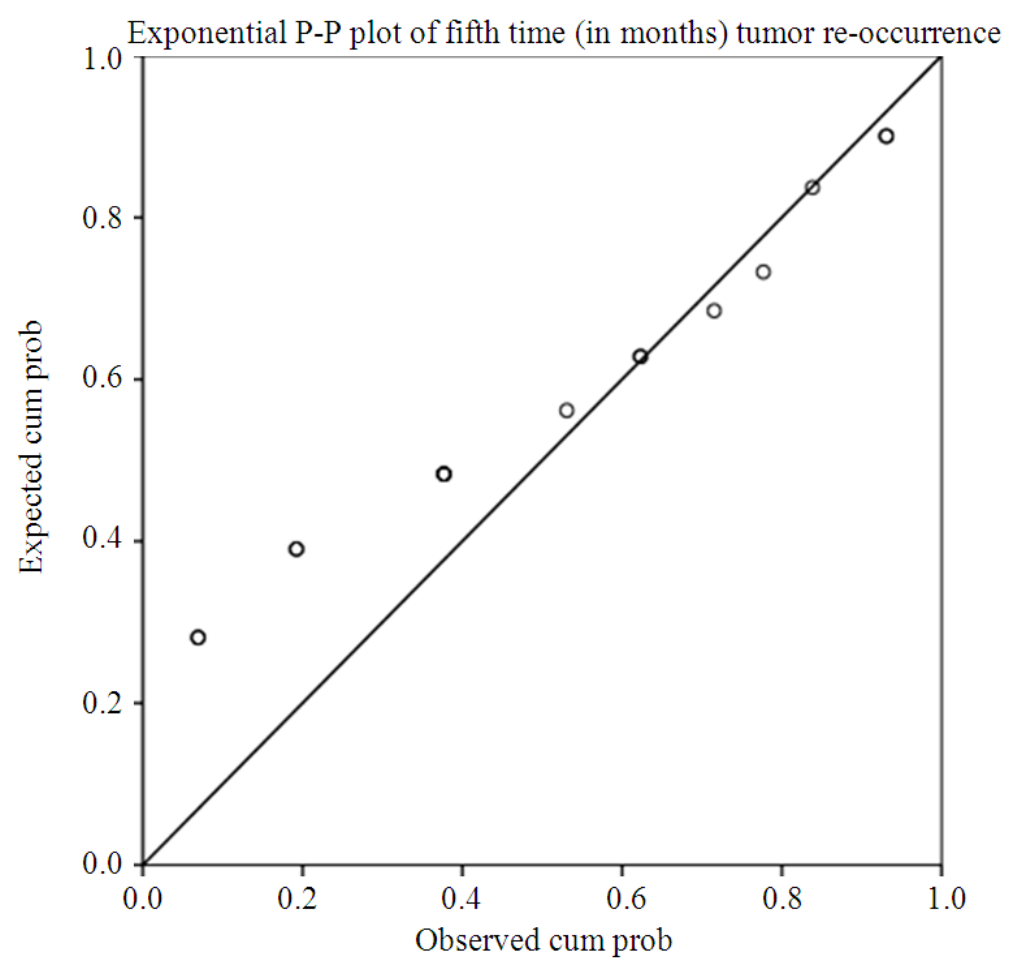

(e)

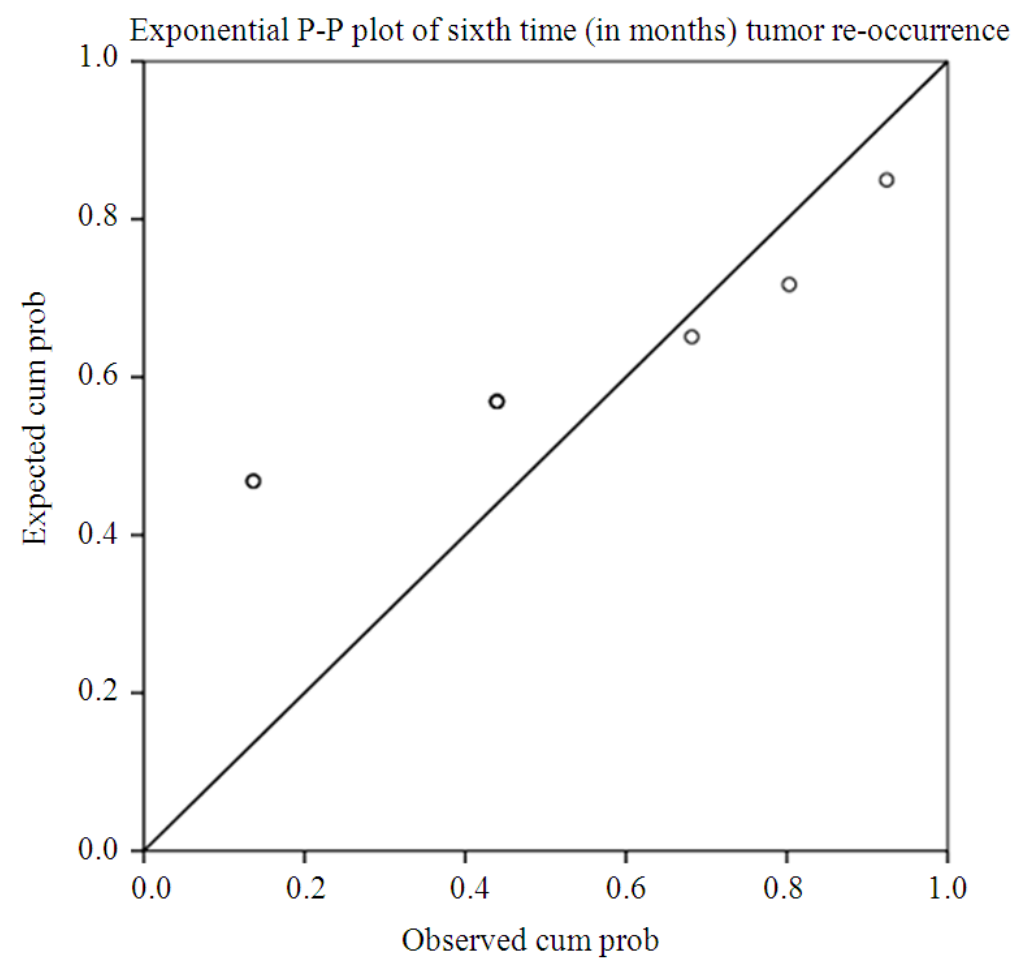

(f) 
Ramalingam Shanmugam / American Medical Journal 4 (1): 43-62, 2013

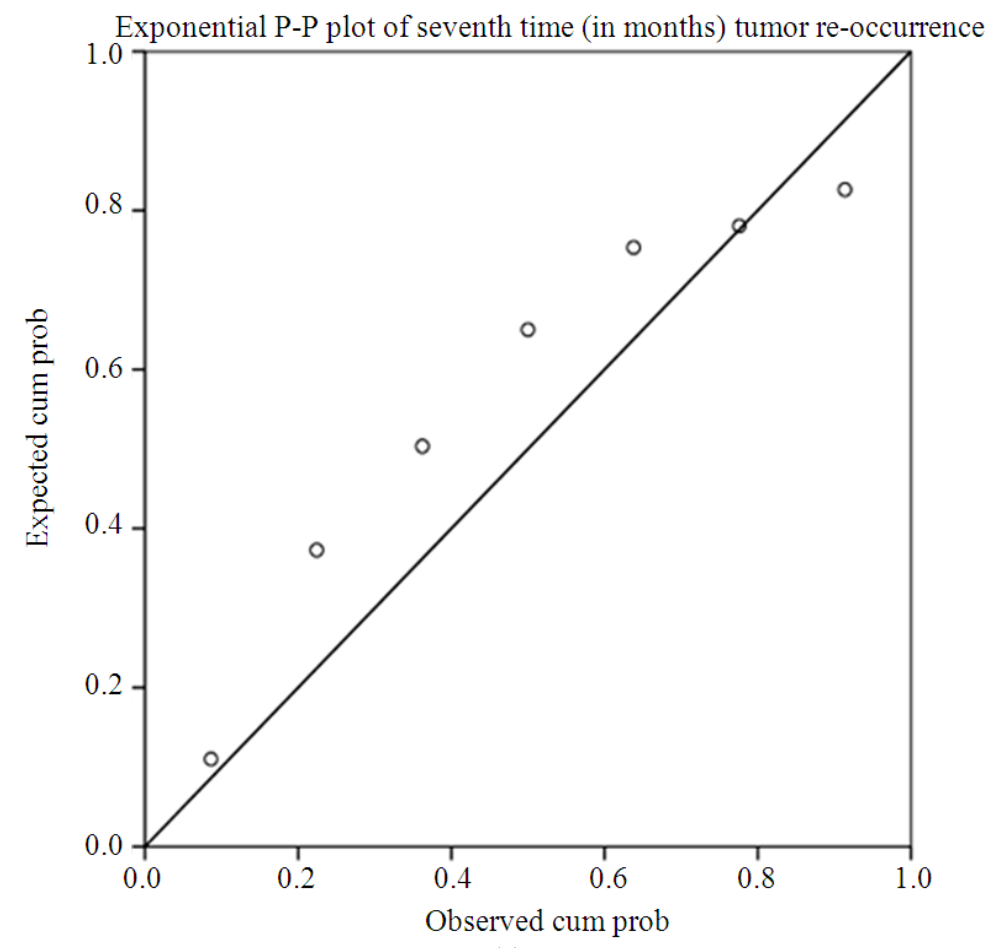

(g)

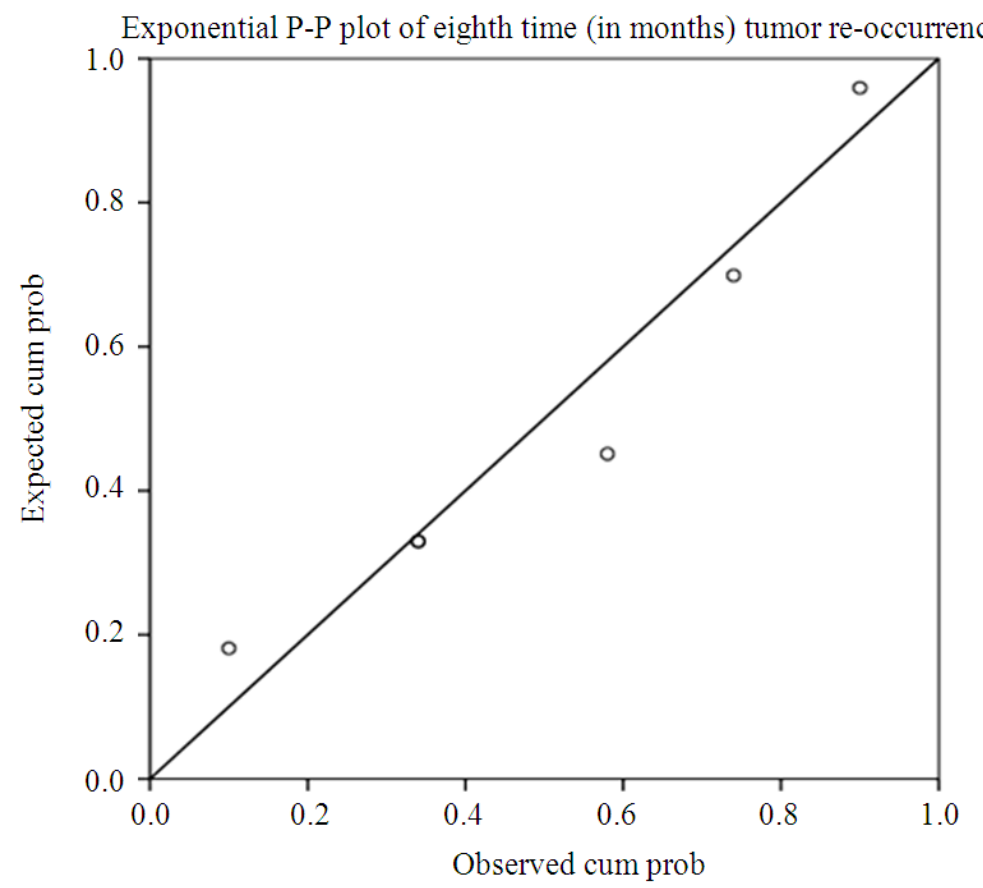

(h)

Fig. 2. (a) First recurrence (b) Second recurrence (c) Third recurrence (d) Fourth recurrence (e) Fifth recurrence (f) Sixth recurrence (g) Seventh recurrence (h) Eighth recurrence 
Ramalingam Shanmugam / American Medical Journal 4 (1): 43-62, 2013

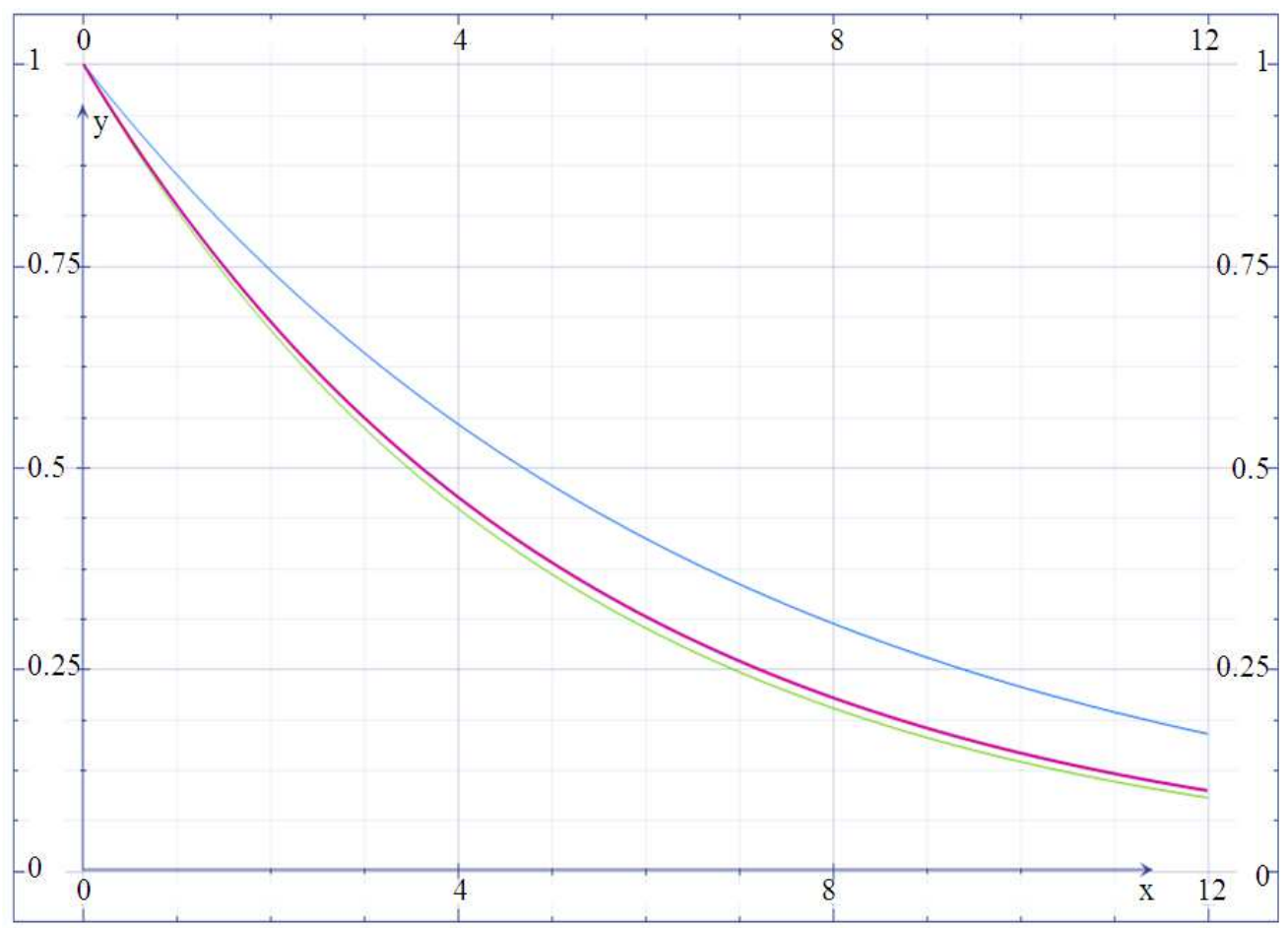

(a)

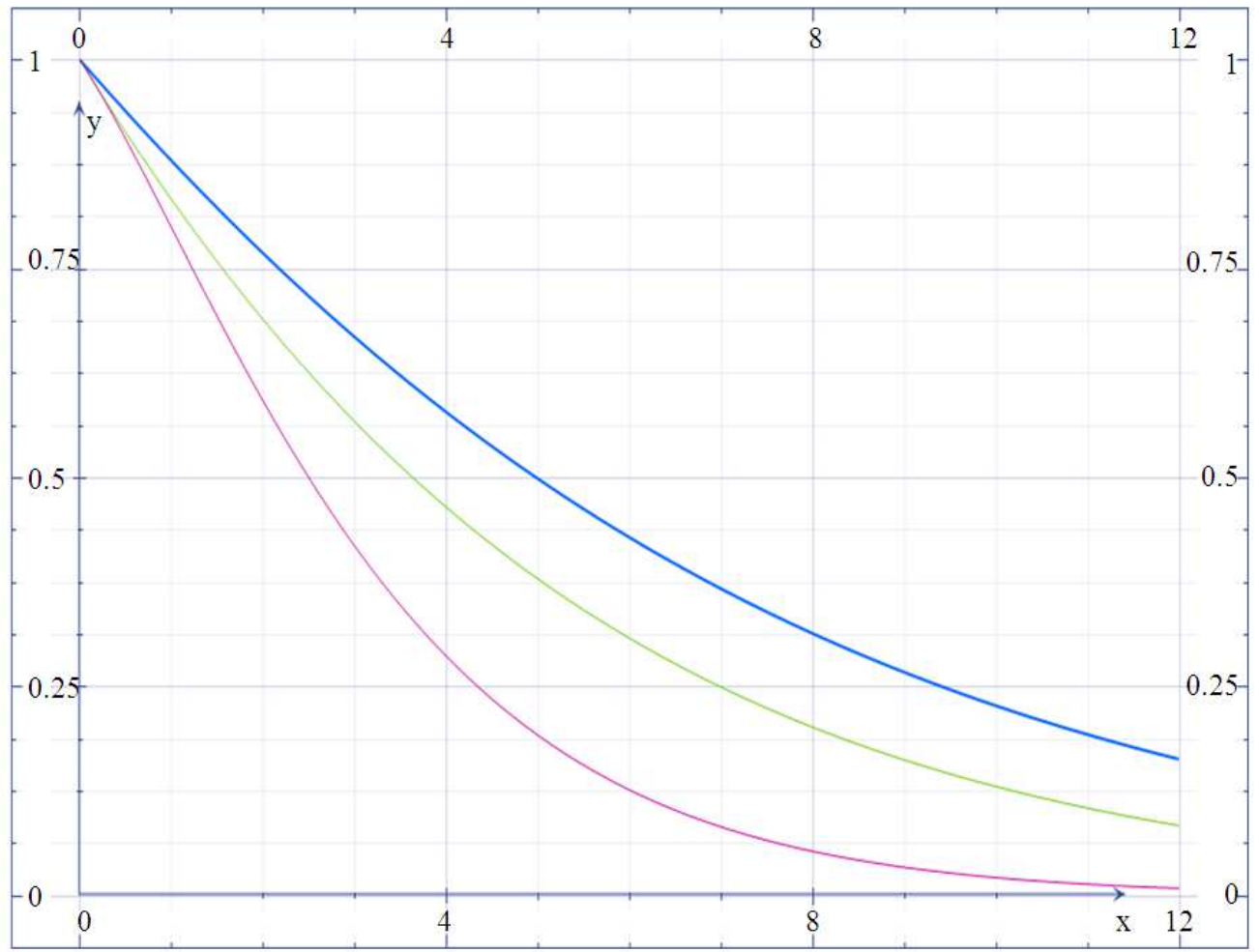

(b) 
Ramalingam Shanmugam / American Medical Journal 4 (1): 43-62, 2013

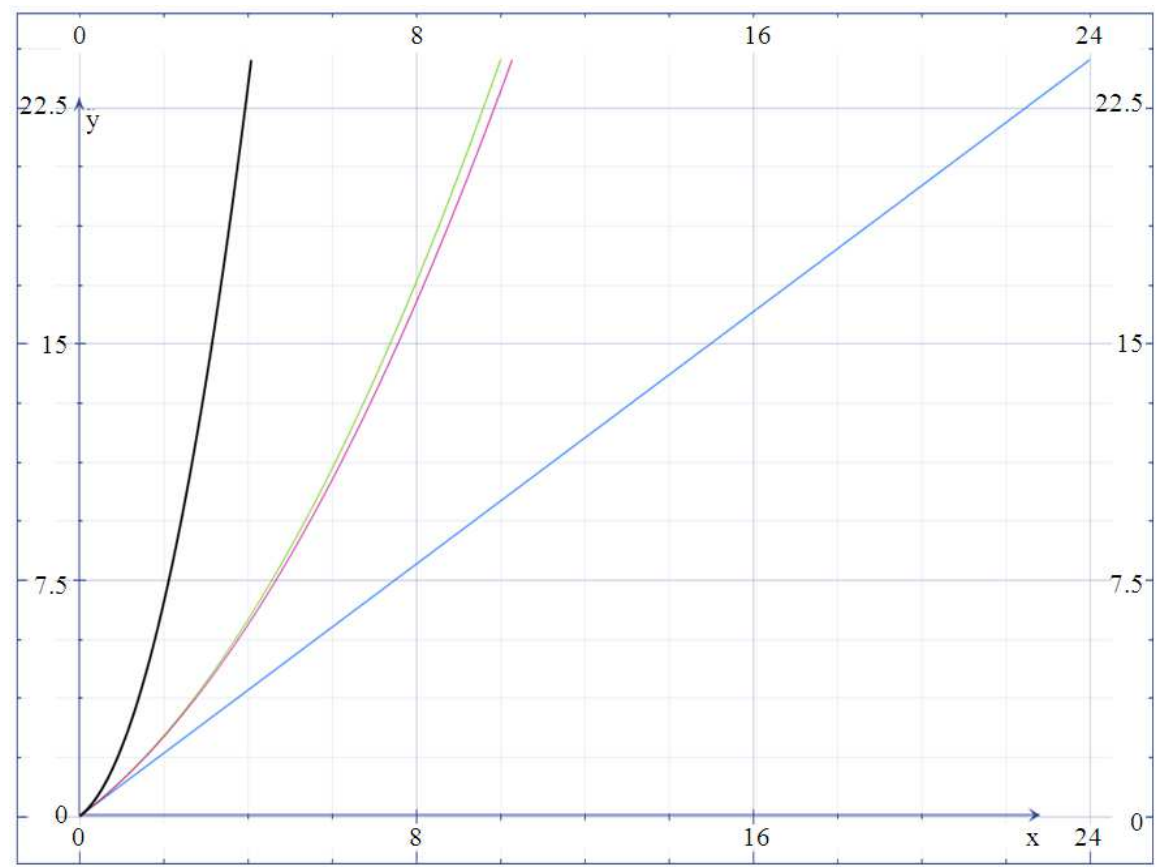

(c)

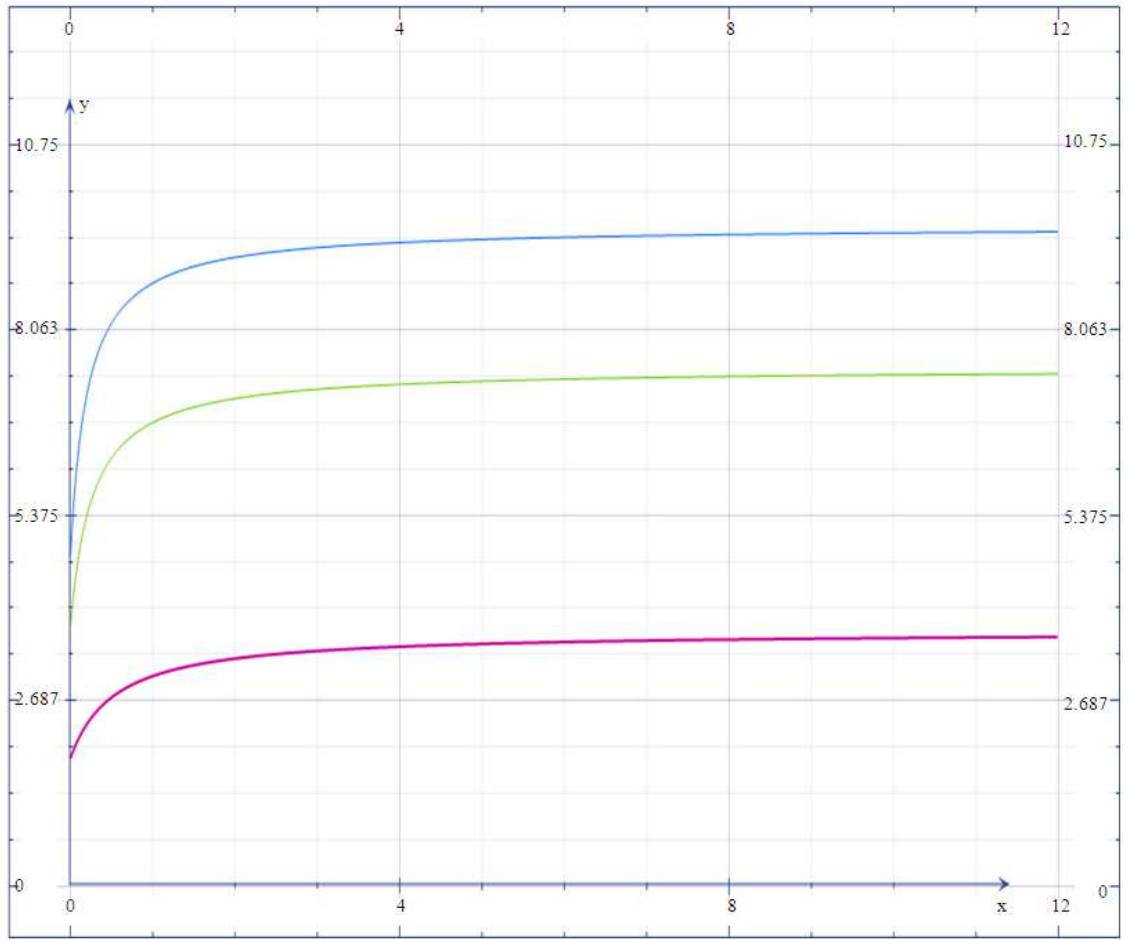

(d)

Fig. 3. (a) Survival function in Equation (5.2) (b) Survival function with (5.1) (c) Recurrence time in terms of $\tau$ when $\hat{\rho}=1.2,0.14$, $0.13,0$ (d) Expected recurrence time in terms of $\rho$ when $\hat{\tau}=4.78,3.75,1.84$ 
The methodology is also illustrated using recurrence times of three groups of bladder cancer patients in Andrews and Herzberg (1985). In the end, some conclusive comments are made.

\subsection{Prolonged Survival Exponential Distribution with Properties}

Lack of memory is desirable feature in discussions of adversarial events. In other situations of favorable outcomes, especially in health mechanism such as prolonging bladder/cancer recurrence time, possessing memory may be adesirable feature. To be specific, suppose that the expected bladder cancerrecurrencetime increases from $\tau$ byan amount $\left[1+\frac{\rho \tau}{1+\rho \tau}\right]$ due to an effective medication, where the prolonging parameter $\rho \geq 0$ signifies the medication's effect. That means the conditional survival probability becomes:

$$
\begin{aligned}
& \operatorname{Pr}[\mathrm{Y}>\mathrm{t}+\mathrm{m} \mid \mathrm{Y}>\mathrm{t}] \\
& =\frac{\operatorname{Pr}[\mathrm{Y}>\mathrm{t}+\mathrm{m}]}{\operatorname{Pr}[\mathrm{Y}>\mathrm{t}]} \\
& =\left[1+\frac{\rho \mathrm{m}}{1+\rho(\tau+\mathrm{t})}\right] \mathrm{e}^{-\mathrm{m} / \tau}
\end{aligned}
$$

In other words, the conditional survivability in Equation (3) is prolonged by an amount prolong $_{(t, m)}=\left[\frac{\rho m}{1+\rho(\tau+t)}\right]$ at a time $t \geq 0$ because of the medication's effect. The memory boost is a unique characteristic of prolonged exponential distribution in Equation (5a).

The unconditional Survival Function (SF) could be captured by a change of variables $\mathrm{t}=0$ and $\mathrm{m}=\mathrm{y}$ in Equation (3). The unconditional SF is:

$$
\begin{aligned}
& \mathrm{S}_{\text {prolExp }}(\mathrm{y} \mid \rho, \tau)=\operatorname{Pr}[\mathrm{Y}>\mathrm{y} \mid \rho, \tau] \\
& =\left[1+\frac{\rho \mathrm{y}}{1+\rho \tau}\right] \mathrm{e}^{-\mathrm{y} / \tau} \\
& \mathrm{y}>0, \tau>0, \rho \geq 0
\end{aligned}
$$

The survival probability, $\mathrm{S}_{\text {pro|Exp }}(\mathrm{y} \mid \rho, \tau)$ in Equation (4) of a cancer patient without a recurrence under a stronger (that is, $\rho \rightarrow \infty$ ) medication's effect is better serving than their counterpart survival probability, $S_{\exp }$ $(y \mid \tau)$ in Equation (1) based on exponential distribution with no role for medication's effect.
Incidentally, the concept of survival function was originated in engineering context to assess the probability for a component to survive a given time without a failure. It is the engineering background in which the nomenclatures like hazard function was created. In the context of treating cancer patients with an intention to postpone a recurrence as much as possible, the survival function in Equation (4) makes more senseas it has a builtin prolonging parameter to reflect the medication's impact.

Now, by differentiating the survival probability in Equation (4) with respect to y, the Probability Density Function (PDF) of the Prolonged Survival Exponential Distribution (PSED) in Equation (5a) below is obtained. The PDF of PSED is:

$$
\begin{aligned}
& f(y \mid \rho, \tau)=\frac{(1+\rho y) e^{-y / \tau}}{\tau(1+\rho \tau)}, \\
& y \geq 0, \tau>0, \rho \geq 0
\end{aligned}
$$

When there is no medication's effect (that is, $\rho=0$ ), the PDF in Equation (5a) reduces to the PDF:

$$
\mathrm{f}(\mathrm{y} \mid \tau)=\mathrm{e}^{-\mathrm{y} / \tau} / \tau, \mathrm{y} \geq 0, \tau>0
$$

Of the exponential distribution as a particular case. An implication is then using exponential distribution for bladder or other cancer reoccurrence time data analysis amounts to preposterously assuming that the patients underwent an ineffective medication and it is a biased data analysis. Instead, shouldn't the patients' data guide the selection of an appropriate model? It is possible that in some instances, the collected data might reject PSED in Equation (5a) in favor of exponential distribution in Equation $(5 \mathrm{~b})$. Details of the model selection between ED in Equation (5b) and PSED in Equation (5a) are discussed in a later part of this section using Wald's likelihood ratio testing criterion.

Now, several features of PSED in Equation (5a) are explored. First, its mean, median and variance are derived and discussed. The mean is in Equation (6a):

$\mu_{\text {mean }}=\mathrm{E}(\mathrm{Y})=\tau\left(1+\frac{\rho \tau}{1+\rho \tau}\right)$

The Taylor series expansion of the survival function $\mathrm{S}_{\text {prolExp }}(\mathrm{y} \mid \rho, \tau)=1-\pi$ yields the $\pi^{\text {th }}$ percentile $\mathrm{y}_{\pi} \approx\left(\frac{\pi \tau}{1+\rho \tau}\right)$. The median is therefore $\mu_{\text {median }} \approx \frac{\tau}{2(1+\rho \tau)}$. The PSED in Equation (5a) is positively skewed because the mean is 
larger than the median since $1+4 \rho \tau>0$. The variance in Equation (6b) is:

$$
\begin{aligned}
& \sigma^{2}=\tau^{2}\left[1+\left(\frac{\rho \tau}{1+\rho \tau}\right)\left(1+\frac{\rho \tau}{1+\rho \tau}\right)\right. \\
& =\tau\left[\tau+\left(\mu_{\text {mean }}-\tau\right) \mu_{\text {mean }}\right]
\end{aligned}
$$

Next, its Hazard Function (HF) in Equation (6c) is derived and explained. The HF for PSED in Equation (5a) is:

$$
\begin{aligned}
& \mathrm{h}(\mathrm{y} \mid \rho, \tau)=\frac{\mathrm{f}(\mathrm{y} \mid \rho, \tau)}{\mathrm{S}_{\text {prolExp }}(\mathrm{y} \mid \rho, \tau)} \\
& =\left[\tau\left(1+\frac{\rho \tau}{1+\rho \mathrm{y}}\right)\right]^{-1}
\end{aligned}
$$

In the context of treating cancer patients, the term "hazard" is probably a misnomenclature, although this popular terminology refers reliability of an electronic component in engineering context. Instantaneously (that is, as $\mathrm{y} \rightarrow 0)$, the HF of PSED (5.1) is $[\tau(1+\rho \tau)]^{-1}$ but it monotonically decreases to zero eventually (that is, when the recurrence time $\mathrm{y} \rightarrow \infty)$. Recall that the $\mathrm{HF}$ of exponential distribution in Equation (5b) is just $\tau^{-1}$ and it is meaningless for cancer recurrences. With a stronger medication's effect (that is, $\rho \rightarrow \infty$ ), the HF stabilizes at $\left[\tau\left(1+\frac{\tau}{1+y}\right)\right]^{-1}$. Of course, the medication's effect should have a cumulative impact. The Cumulative Hazard Function (CHF) of the PSED in Equation (5a) reflects it in Equation (7) below:

$$
\begin{aligned}
& \operatorname{CHF}(y \mid \rho, \tau)=\int_{0}^{y} \tau^{-1}\left[1+\frac{\rho \tau}{1+\rho \mathrm{u}}\right]^{-1} \mathrm{du} \\
& =\frac{\mathrm{y}}{\tau}+\ln \left(1-\frac{\rho \mathrm{y}}{1+\rho[\mathrm{y}+\tau]}\right) \\
& \approx \frac{\mathrm{y}}{\tau}-\left(\frac{\rho \mathrm{y}}{1+\rho[\mathrm{y}+\tau]}\right)^{2}
\end{aligned}
$$

The CHF converges asymptotically to $\frac{\mathrm{y}}{\tau}, 0$ or $\infty$ under no medication's effect (that is, $\rho=0$ ), initially (that is, $\mathrm{y}=0$ ) and eventually (that is, $\mathrm{y}=\infty$ ) respectively. In the context of treating cancer with a medical aim to help the patient keep going on without a recurrence, it is appropriate to think of the function $\mathrm{CHF}$ in Equation (7) as the cumulative impact of the medication's effect. On the assumption that a patient survived without a recurrence of cancer at a current time, the chance for $\mathrm{s} / \mathrm{he}$ to have an extra residual time, without a recurrences $\left[1+\frac{\rho u}{\tau+\rho(y+1)}\right] e^{-u / \tau} S_{\text {prolExp }}(y \mid \rho, \tau)$.

Furthermore, the Mean Residual Life Function (MRLF) captures how much time (unconditionally) is left without a recurrence on the average. In our discussion of cancer patients with amedication's effect, the MRLF of PSED in Equation (5a) is:

$$
\begin{aligned}
& \operatorname{MRLF}(y \mid \rho, \tau) \\
& =\int_{0}^{\infty} \frac{S_{\text {prolExp }}(y+u \mid \rho, \tau)}{S_{\text {prolExp }}(y \mid \rho, \tau)} d u \\
& =\int_{0}^{\infty}\left[1+\frac{\rho u}{1+\rho(\tau+y)}\right] e^{-u / \tau} d u \\
& =1+\frac{\rho \tau^{2}}{1+\rho(y+\tau)}
\end{aligned}
$$

When the patient gets no medication's effect (that is, $\rho=0$ ), the MRLF in Equation (8) gives the baseline amount but it increases when the patient receives an effective medication's effect. The gained time, unconditionally speaking, with no cancer recurrence is $\frac{\rho \tau^{2}}{1+\rho(y+\tau)}$. This gained time stabilizes at $\frac{\tau^{2}}{(y+\tau)}$ under a stronger medication's effect (that is, $\rho \rightarrow \infty$ ).

In some instances, once a patient has survived a time $\mathrm{d}$, the medical team might wonder how much additional time exists? Conditionally speaking, on the average for the patient to experience no cancer recurrence can be addressed as follows. This concept is the Mean Excess Time (MET). Panjer (2006) for details about its importance in making decisions. For PSED in Equation (5a), the MET is:

$$
\begin{aligned}
& E(Y-d \mid Y>d)=\frac{\int_{d}^{\infty} S_{\text {prolexp }}(y \mid \rho, \tau) d y}{S_{\text {prolExp }}(d \mid \rho, \tau)} \\
& =\frac{\int_{d}^{\infty}\left(1+\frac{\rho y}{1+\rho \tau}\right) e^{-\frac{y}{\tau}} d y}{\left(1+\frac{\rho d}{1+\rho \tau}\right) e^{-\frac{d}{\tau}}} \\
& =\tau\left(1+\frac{\rho \tau}{1+\rho[\tau+d]}\right)
\end{aligned}
$$

The MET in Equation (9) asymptotically approaches $\tau$ or $\tau\left(1+\frac{\tau}{\tau+d}\right)$ as $\rho=0$ or $\rho=\infty$ respectively. 
However, in the initial time (that is, $d=0$ ) or eventually (that is, $d=\infty$ ), the MET in Equation (9) is at an asymptotic level $\tau\left(1+\frac{\rho \tau}{1+\rho \tau}\right)$ or $\tau$ respectively.

After the end of treatment time, the patients are advised to keep in contact with the medical team. Because of the patient's migration to another location or the hospital's administrative inability, such a follow up unfortunately terminates. The duration between the end of treatment time and the last contact time is called alimited follow-up window time and it is indicated by $\mathrm{Y} \wedge \mathrm{u}=\mathrm{Y}$ if $\mathrm{Y}<\mathrm{u}$ and $\mathrm{u}$ if $\mathrm{Y} \geq \mathrm{u}$. See Blumenfeld (2010) for details about the role of follow-up window time to make up operational decisions. The Expected Limited Follow-Up Window Time (ELFWT) for PSED in Equation (5a) is:

$$
\begin{aligned}
& E(Y \wedge u) \\
& =\int_{0}^{\infty} y f(y \mid \rho, \tau) d y+u S_{\text {prolExp }}(y \mid \rho, \tau) \\
& =\int_{0}^{\infty} \frac{y}{\tau(1+\rho \tau)}\left(1+\frac{\rho y}{1+\rho \tau}\right) e^{-\frac{y}{\tau}} d y \\
& \quad+u\left(1+\frac{\rho u}{1+\rho \tau}\right) e^{-\frac{u}{\tau}} \\
& =\tau\left(1+\frac{\rho \tau}{1+\rho \tau}\right)+u\left(1+\frac{\rho u}{1+\rho \tau}\right) e^{-\frac{u}{\tau}} .
\end{aligned}
$$

The ELFWT is $\tau+\mathrm{ue}^{-\frac{\mathrm{u}}{\tau}}$ for exponential distribution in Equation (5a) with a substitution of $\rho=0$ in Equation (10). Understandably, the ELFWT in Equation (10) increases once the medication's effect is effective (that is, as $\rho>0$ ). Of course, the ELFWT varies from a patient to another. Hence, the spread of the follow-up window time in Equation (11) is necessary to understand the heterogeneity among the patientsand it is captured by its variance of prolonged exponential distribution in Equation (5a). It is:

$$
\begin{aligned}
& \operatorname{var}(Y \wedge u)=\tau^{2}\left[1+\left(\frac{\rho \tau}{1+\rho \tau}\right)\left(1+\frac{\rho \tau}{1+\rho \tau}\right)\right] \\
& +u^{2}\left(1+\frac{\rho u}{1+\rho \tau}\right) e^{-\frac{u}{\tau}}\left[1-\left(1+\frac{\rho u}{1+\rho \tau}\right) e^{-\frac{u}{\tau}}\right] \\
& -2 \tau u\left(1+\frac{\rho \tau}{1+\rho \tau}\right)\left(1+\frac{\rho u}{1+\rho \tau}\right) e^{-\frac{u}{\tau}}
\end{aligned}
$$

Another useful concept is the risk for a cancer patient to experience a recurrence given the patient has survived a time $y_{p}$. This concept is called Total Value at Risk $\left(\mathrm{TVaR}_{\mathrm{p}}\right)$. With the PSED in Equation $(5 \mathrm{a})$, the $\mathrm{TVaR}_{\mathrm{p}}$ is:

$$
\begin{aligned}
& \operatorname{TVaR}_{p}(Y)=E\left(Y \mid Y>y_{p}\right) \\
& =\frac{\int_{y_{p}}^{\infty} y f(y \mid \rho, \tau) d y}{S_{\text {prolExp }}\left(y_{p} \mid \rho, \tau\right)} \\
& =\frac{\int_{p}^{1} \operatorname{VaR}_{u}(u) d u}{1-p} \\
& =y_{p}+\frac{\int_{y_{p}}^{\infty}\left(y-y_{p}\right) f(y \mid \rho, \tau) d y}{S_{\text {prolExp }}\left(y_{p} \mid \rho, \tau\right)} \\
& \approx \tau(1+\rho \tau)\left[\left(1+\frac{\rho y_{p}}{1+\rho \tau}\right) e^{-\left(\frac{y_{p}}{\tau}\right)}\right]^{2}
\end{aligned}
$$

The $\mathrm{TVaR}_{\mathrm{p}}$ in Equation (12) is $\tau(1+\rho \tau)$ initially (that is, $y_{p}=0$ ) and zero eventually (that is, $y_{p}=\infty$ ). However, the $\mathrm{TVaR}_{\mathrm{p}}$ is $\tau \mathrm{e}^{-\frac{2 y_{\mathrm{p}}}{\tau}}$ for exponential distribution in Equation (5b) by substituting $\rho=0$ in Equation (12).

In some patients, the cancer recurrence might be quicker than its usual time. Such patients receive special attention of the medical team. To such patients, the medical team might decide to apply additional remedial actions. To decide on such a remedial action, the PDF $f_{Y(1)}(u)$ and its expected value $E_{Y(1)}(u)$ of the minimal order statistic $\mathrm{Y}_{(1)}$ are useful. That is:

$$
\begin{aligned}
& \mathrm{f}_{\mathrm{Y}_{(1)}}(\mathrm{u})=\mathrm{n}\left[\mathrm{S}_{\text {prolExp }}(\mathrm{u} \mid \rho, \tau)\right]^{\mathrm{n}-1} \mathrm{f}(\mathrm{u}) \\
& \approx \frac{\mathrm{n}^{2}[1+(\mathrm{n}-1+\mathrm{u}-\tau) \rho] \mathrm{e}^{-\mathrm{nu} / \tau}}{\tau[\mathrm{n}+(\mathrm{n}-1)(\mathrm{n}-\tau) \rho]} ; \mathrm{u} \geq 0
\end{aligned}
$$

For prolonged exponential distribution in Equation (5a) with mean in Equation (13):

$$
\begin{aligned}
& E(U)=\int_{0}^{\infty} f_{Y_{(1)}}(u) d u \\
& =\frac{\tau}{n}\left[1+\frac{\rho \tau}{n+(n-1)(n-\tau) \rho}\right]
\end{aligned}
$$

Which is $\tau / \mathrm{n}$ under no medication's effect (that is, when $\rho=0$ ) but increases to a stable amount:

$$
\tau\left[1+\frac{\tau}{n+(n-1)(n-\tau)]}\right] / n
$$


Under most effective medication's effect (that is, $\rho \rightarrow \infty$ ).

Furthermore, when a patient exhibits consequently two quicker cancer recurrences, it is a sign of deterioration. Such patients are of concern to the medical team. This deterioration is detected using Markov-Chain property. The Markov-chain property with PSED in Equation (5a) is echoed in the PDF $f_{Y(2)=u+w \mid Y(1)}=u(w)$ of the second order statistic $Y_{(2)}=v=u+w$ given the first order statistic $\mathrm{Y}_{(1)}=\mathrm{u}$ where $\mathrm{w} \geq 0$. It is:

$$
\begin{aligned}
& \mathrm{f}_{\mathrm{Y}_{(2)}=\mathrm{u}+\mathrm{w} \mid \mathrm{Y}_{(1)}=\mathrm{u}}(\mathrm{w}) \\
& =(\mathrm{n}-1)\left\{\frac{\mathrm{S}_{\text {prolExp }}(\mathrm{v} \mid \rho, \tau)}{\mathrm{S}_{\text {prolExp }}(\mathrm{u} \mid \rho, \tau)}\right\}^{\mathrm{n}-2} \frac{\mathrm{f}(\mathrm{v})}{\mathrm{f}(\mathrm{u})} \\
& \approx\left(\frac{\mathrm{n}-1}{\tau}\right) \frac{[1+(\mathrm{n}-2) \rho \mathrm{w}]}{[1+(\mathrm{n}-2) \rho]} \mathrm{e}^{-(\mathrm{n}-1) \mathrm{w} / \tau}
\end{aligned}
$$

Whose mean is:

$$
\begin{aligned}
& \mathrm{E}_{\text {Markovian }}(\mathrm{w} \mid \mathrm{u}) \\
& =\int_{0}^{\infty} \mathrm{wf}_{\mathrm{Y}_{(2)}=\mathrm{u}+\mathrm{w} \mid \mathrm{Y}_{(1)}=\mathrm{u}}(\mathrm{w}) \mathrm{dw} \\
& =\frac{\tau}{(\mathrm{n}-1)^{2}}\left[1+\frac{(\mathrm{n}-2)\{1+(2 \rho \tau-1) \rho\}}{1+(\mathrm{n}-2) \rho}\right]
\end{aligned}
$$

With no medication's effect (that is, $\rho=0$ ), the PDF in Equation (14) reduces to:

$$
\mathrm{f}_{\mathrm{Y}_{(2)}=\mathrm{u}+\mathrm{w} \mid \mathrm{Y}_{(1)}=\mathrm{u}}(\mathrm{w}) \approx\left(\frac{\mathrm{n}-1}{\tau}\right) \mathrm{e}^{-(\mathrm{n}-1) \mathrm{w} / \tau}
$$

Whose mean is:

$$
\mathrm{E}_{\text {Markovian }}(\mathrm{w} \mid \mathrm{u})=\frac{\tau}{(\mathrm{n}-1)}
$$

Corresponding to exponential distribution in Equation (5b) by substituting $\rho=0$. In this scenario of quicker cancer recurrence than the usual time, medical team would get alerted to seek a medical urgency. Consequently, the chance for the remaining time to be without a cancer recurrence changes and it is captured by the equilibrium distribution. Using PSED in Equation (5b), the equilibrium distribution in Equation (15) is:

$$
\begin{aligned}
& \mathrm{H}_{\text {MBED }}(\mathrm{y} \mid \rho, \tau) \\
& =\frac{\int_{0}^{\mathrm{y}} \mathrm{S}_{\text {prolExp }}(\mathrm{u} \mid \rho, \tau) \mathrm{du}}{\int_{0}^{\infty} \mathrm{S}_{\text {prolExp }}(\mathrm{u} \mid \rho, \tau) \mathrm{du}} \\
& =\tau\left(1-\mathrm{e}^{-\mathrm{y} / \tau}\right)+\frac{\rho \tau}{(1+\rho \tau)}\left\{1-\left(1+\frac{\mathrm{y}}{\tau}\right) \mathrm{e}^{-\mathrm{y} / \tau}\right\}
\end{aligned}
$$

With an intensity rate:

$$
\begin{aligned}
& \frac{\mathrm{S}_{\text {prolExp }}(\mathrm{u} \mid \rho, \tau)}{\mathrm{H}_{\mathrm{MBED}}(\mathrm{y} \mid \rho, \tau) \int_{0}^{\infty} \mathrm{S}_{\text {prolExp }}(\mathrm{u} \mid \rho, \tau) \mathrm{du}} \\
& =\frac{[1+\rho(\mathrm{y}+\tau)]}{\tau\left[(1+\rho \tau)\left(\mathrm{e}^{\mathrm{y} / \tau}-1\right)+\rho \tau\left\{\mathrm{e}^{\mathrm{y} / \tau}-\left(1+\frac{\mathrm{y}}{\tau}\right\}\right]\right.}
\end{aligned}
$$

For exponential distribution in Equation (5b), the equilibrium distribution reduces to $\tau\left(1-\mathrm{e}^{-\mathrm{y} / \tau}\right)$ with intensity rate $\left[\tau\left(e^{y / \tau}-1\right)\right]^{-1}$ by substitution of $\rho=0$ in Equation (16).

To advance the medical knowledge, the medical team looks into patient records with a quicker or longer cancer recurrence. Ahsanullah (1995; 1978) for concepts and formulas to understand the role of record values in data analysis. The PDF $f_{\text {lange-record }}(v)$ of the Longer Recorded Time (LRT), $v$ for PSED in Equation (5a) is:

$$
\begin{aligned}
& \mathrm{f}_{\text {record-longer }}(\mathrm{v}) \\
& =\frac{\left\{-\ln \mathrm{S}_{\text {prolExp }}(\mathrm{v} \mid \rho, \tau)\right\}^{\mathrm{n}} \mathrm{f}(\mathrm{v} \mid \rho, \tau)}{\mathrm{n} !} \\
& =\frac{[1+\rho(\mathrm{v}-\tau)] \mathrm{v}^{\mathrm{n}} \mathrm{e}^{-\mathrm{v} / \tau}}{\mathrm{n} ! \tau^{\mathrm{n}+1}(1+\mathrm{n} \rho \tau)} ; \mathrm{v} \geq 0
\end{aligned}
$$

From Equation (17), the mean and variance of the longer-record recurrence times are respectively:

$$
\mathrm{E}_{\text {longer-record }}(\mathrm{V})=(\mathrm{n}+1) \tau\left[1+\frac{\rho \tau}{1+\mathrm{n} \rho \tau}\right]
$$

And:

$$
\begin{aligned}
& \operatorname{Var}_{\text {longer-record }}(V) \\
& =(n+1) \tau^{2}\left[1+\frac{\rho \tau(2+\{n-1\} \rho \tau)}{(1+n \rho \tau)(1+n \rho \tau)}\right]
\end{aligned}
$$

With these results, we may pose a question: When is alonger-recordcancer occurrence significant? The longerrecord recurrence time is significant when the $\mathrm{p}$-value:

$$
1-\Phi\left(\frac{\left.\mathrm{v}-(\mathrm{n}+1) \tau\left[1+\frac{\rho \tau}{1+\mathrm{n} \rho \tau}\right]\right]}{\sqrt{(\mathrm{n}+1) \tau^{2}\left[1+\left(\frac{\rho \tau}{1+\mathrm{n} \rho \tau}\right)\left(\frac{2+\{\mathrm{n}-1\} \rho \tau}{1+\mathrm{n} \rho \tau}\right)\right]}}\right)
$$

Is small, where $\Phi(\mathrm{z})$ is the cumulative standard normal probability. 
Likewise, the medical team is concerned about the patients with a quicker-record recurrence time also. The $\mathrm{PDF} \mathrm{f}_{\text {quicker-rcord }}(\mathrm{u})$ of the quicker recorded time, $\mathrm{U}$ is:

$\mathrm{f}_{\text {quic ker-record }}(\mathrm{u})$

$=\frac{[1+\{(\mathrm{n}-2) \tau+\mathrm{u}\} \rho] \mathrm{ue}^{-\mathrm{u} / \tau}}{\tau^{2}(1+\mathrm{n} \rho \tau)} ; \mathrm{u} \geq 0$

From Equation (20), the mean and variance of the quicker-record recurrence times are respectively:

$$
\mathrm{E}_{\text {quicker-record }}(\mathrm{U})=2 \tau\left(1+\frac{\rho \tau}{1+\mathrm{n} \rho \tau}\right)
$$

And:

$$
\begin{aligned}
& \operatorname{Var}_{\text {quicker-record }}(\mathrm{U}) \\
& =2 \tau^{2}\left[1+\left(\frac{2 \rho \tau}{1+\mathrm{n} \rho \tau}\right)\left(\frac{1+\{\mathrm{n}-1\} \rho \tau}{1+\mathrm{n} \rho \tau}\right)\right]
\end{aligned}
$$

We may pose another question: Is a quicker record cancer occurrence significant? It is so if the p-value:

$$
\Phi\left(\frac{U-2 \tau\left(1+\frac{\rho \tau}{1+\mathrm{n} \rho \tau}\right)}{\sqrt{2 \tau^{2}\left[1+\left(\frac{2 \rho \tau}{1+\mathrm{n} \rho \tau}\right)\left(\frac{1+\{\mathrm{n}-1\} \rho \tau}{1+\mathrm{n} \rho \tau}\right)\right]}}\right)
$$

Is small with this knowledge about the quickerrecord, $\mathrm{U}$ and longer-record, $\mathrm{V}$ of bladder cancer recurrence times, their correlation $r_{u, v}$ is useful and it reveals medically valuable information. To compute it, the joint PDF $\mathrm{f}_{\text {quicker-record,longer-record }}(\mathrm{u}, \mathrm{v})$ of the quicker-recordandlonger-record cancer recurrence is necessary and it is:

$$
\begin{aligned}
& \mathrm{f}_{\text {record-quic ker, record-longer }}(\mathrm{u}, \mathrm{v}) \\
& =\left\{\frac{-\ln \mathrm{S}_{\text {prolExp }}(\mathrm{u} \mid \rho, \tau)}{(\mathrm{n}-2) !}\right\} \\
& \left\{-\ln \left(\frac{\mathrm{S}_{\text {prolExp }}(\mathrm{v} \mid \rho, \tau)}{\mathrm{S}_{\text {prolExp }}(\mathrm{u} \mid \rho, \tau)}\right\}^{(\mathrm{n}-2)} \frac{\mathrm{f}(\mathrm{v}) \mathrm{f}(\mathrm{u})}{\mathrm{S}(\mathrm{u})}\right. \\
& =\frac{[1+\rho(\mathrm{v}-\tau)] \mathrm{u}(\mathrm{v}-\mathrm{u})^{\mathrm{n}-2} \mathrm{e}^{-\mathrm{v} / \tau}}{(\mathrm{n}-2) ! \tau^{\mathrm{n}+1}(1+\mathrm{n} \rho \tau)}
\end{aligned}
$$

Of course, their correlation $r_{u, v}$ captures their linear relationship. It is feasible to project the next longerrecord cancer recurrence based on an observedquicker- recordcancer recurrence time using regression concept. For this purpose, from Equation (23), the product moment is found and it is:

$$
\begin{aligned}
& E(U V) \\
& =\int_{u}^{\infty} \int_{v=u}^{\infty} f_{\text {lower-record, upper-record }}(u, v) d v d u \\
& =2(n+2) \tau^{2}\left(1+\frac{2 \rho \tau}{1+n \rho \tau}\right)
\end{aligned}
$$

Hence, their correlation is:

$$
\begin{aligned}
& r_{u, v}=\operatorname{corr}(U, V) \\
& =\sqrt{\left.\frac{2\left[1-\frac{(n-1) \rho^{2} \tau^{2}}{(1+n \rho \tau)^{2}+2 \rho \tau\{1+(n-1) \rho \tau\}}\right]}{(n+1)}\right]}
\end{aligned}
$$

The regression Equation, based on the correlation in Equation (24), the means in in Equations (18), (21) and variance in Equations (19), (22) of both quicker-record and longer-record recurrence times, is in Equation (25):

$$
\begin{aligned}
& \hat{\mathrm{Y}}_{\text {next-longer-record-recurrence }} \\
& =r_{\mathrm{uv}} \sqrt{\frac{\mathrm{var}_{\text {longer-record }}(\mathrm{v})}{\mathrm{var}_{\text {quic ker-record }}(\mathrm{u})}} \mathrm{Y}_{\text {quic ker-record }} \\
& +\overline{\mathrm{Y}}_{\text {recurrences }}\left(1-\mathrm{r}_{\mathrm{uv}} \sqrt{\left.\frac{\mathrm{var}_{\text {longer-record }}(\mathrm{v})}{\mathrm{var}_{\text {quic ker-record }}(\mathrm{u})}\right)}\right.
\end{aligned}
$$

Now, to estimate the parameters of prolonged exponential distribution in Equation (5a), consider a random sample $\mathrm{y}_{1}, \mathrm{y}_{2}, \ldots \mathrm{y}_{\mathrm{n}}$ from prolonged exponential distribution in Equation (5a). The maximum likelihood method is selected over other methods because it possesses invariance property. The log likelihood function, In L (.) for prolonged exponential distribution in Equation (5a) is:

$$
\begin{aligned}
& \ln L\left(y_{1}, y_{2}, \ldots . ., y_{n}\right) \\
& =n \rho(\bar{y}-\tau)+\frac{\rho^{2}}{2}\left(n \tau^{2}-\sum_{i=1}^{n} y_{i}^{2}\right) \\
& -\frac{n \bar{y}}{\tau}-n \ln \tau
\end{aligned}
$$

Then, the score functions $\partial_{\tau} \operatorname{In} \mathrm{L}\left(\mathrm{y}_{1}, \mathrm{y}_{2}, \ldots \mathrm{y}_{\mathrm{n}}\right)$ and $\partial_{\rho}$ In $\mathrm{L}\left(\mathrm{y}_{1}, \mathrm{y}_{2}, \ldots, \mathrm{y}_{\mathrm{n}}\right)$ are derivative of the log likelihood function in Equation (26) with respect to the parameter $\tau$ and $\rho$ respectively, where $\partial_{z}$ denotes the derivative. Equating the score functions to zero and solving them 
simultaneously with their Taylor series expansions provides the Maximum Likelihood Estimators (MLE):

$\hat{\tau}=\frac{s^{2}}{\bar{y}}$

And:

$\hat{\rho}=\left|\frac{\bar{y}}{\mathrm{~s}^{2}}\left[\left(\frac{\bar{y}^{2}}{\mathrm{~s}^{2}}-1\right)^{-1}-1\right]^{-1}\right|$

When $\mathrm{s}^{2}=\overline{\mathrm{y}}^{2}$, notice that the MLE in Equations (28) and (27) reduce to $\hat{\rho}=0$ and $\hat{\tau}_{0}=\bar{y}$ which is the MLE of parameter in exponential distribution in Equation (5b).

In a clinical situation, the medical team might ponder over a question: Is the MLE of $\hat{\rho}$ indicative of a significantly effective medication's effect in the sense of prolonging the cancer recurrence in a patient? An answer to this question requires formulating a hypothesis testing methodology. For this purpose, the Wald (1943) likelihood ratio approach is resorted. The Wald's approach is a powerful methodology and is summarized below. The null hypothesis $\mathrm{H}_{0}: \rho=0$ could be rejected in favor of an alternative hypothesis $H_{1}: \rho=\rho^{*} \neq 0$ using log-likelihood ratio:

$$
\begin{aligned}
& -\ln \Lambda_{\rho=0}=\ln \mathrm{L}(\hat{\rho}, \hat{\tau})-\ln \mathrm{L}\left(\rho=0, \hat{\tau}_{0}\right) \\
& =\mathrm{n}\left|\left(1-\frac{\overline{\mathrm{y}}^{2}}{\mathrm{~s}^{2}}\right)\left\{1+\frac{1+\left(1-\frac{\overline{\mathrm{y}}^{2}}{\mathrm{~s}^{2}}\right)^{2}}{1+\left(1-\frac{\overline{\mathrm{y}}^{2}}{\mathrm{~s}^{2}}\right)}\right\}\right|
\end{aligned}
$$

Which follows a non-central chi-squared distribution with one degrees of freedom (df) and the non-centrality parameter $\hat{\delta}_{\rho=0}=\hat{\rho} / \operatorname{var}(\hat{\rho})$, where $\operatorname{var}(\hat{\rho})$ is the MLE of diagonal element in the inverse of the variancecovariance matrix of the MLEs. Stuart and Ord (1994) for definition and properties of the non-central chi squared distribution. Recall that the variance-covariance matrix of the MLE of the parameters is the inverse of the information matrix:

$$
\mathrm{I}=\left[\begin{array}{ll}
\mathrm{a} & \mathrm{b} \\
\mathrm{b} & \mathrm{c}
\end{array}\right]=\left[\begin{array}{ll}
-\mathrm{E}\left(\partial_{\tau \tau}^{2} \ln \mathrm{L}\right) & -\mathrm{E}\left(\partial_{\rho \tau}^{2} \ln \mathrm{L}\right) \\
-\mathrm{E}\left(\partial_{\tau \rho}^{2} \ln \mathrm{L}\right) & -\mathrm{E}\left(\partial_{\rho \rho}^{2} \ln \mathrm{L}\right)
\end{array}\right]
$$

Where:

$$
\begin{aligned}
& \mathrm{a}=-\mathrm{E}\left(\partial_{\tau \tau}^{2} \ln \mathrm{L}\right) \approx \frac{\mathrm{n}(1+\rho \tau)}{\tau^{2}} \\
& \mathrm{~b}=-\mathrm{E}\left(\partial_{\rho \tau}^{2} \ln \mathrm{L}\right)=\mathrm{n}(1-2 \rho \tau)
\end{aligned}
$$

And:

$$
c=-E\left(\partial_{\rho \rho}^{2} \ln L\right)=n \tau^{2}(1+6 \rho \tau)
$$

The determinant of the matrix I is:

$$
\mathrm{D}=|\mathrm{I}|=\mathrm{ac}-\mathrm{b}^{2}=\mathrm{n}^{2} \rho \tau(1+2 \rho \tau)
$$

And $\operatorname{var}(\hat{\rho})=1 / n \hat{\rho} \hat{\tau}^{3}$. Hence, under null hypothesis, $\mathrm{H}_{0}: \quad \rho=0, \quad$ note that $\hat{\tau}=\bar{y} \quad$ and $\hat{\delta}_{\rho=0}=\hat{\rho} / \operatorname{vâr}\left(\hat{\rho} \mid \mathrm{H}_{0}\right)=\left(\frac{\bar{y}}{\mathrm{~s}^{2}}\right)^{3} / \mathrm{n}$. It is known that the noncentral chi squared distribution with one df and noncentrality parameter $\delta$ approximately follows $\left(1+\frac{\delta}{1+\delta}\right)$ times a central chi squared distribution with $\frac{(1+\delta)^{2}}{(1+2 \delta)} d f$ (Stuart and Ord, 1994) for details of this equivalence.

This means that the null hypothesis $\mathrm{H}_{0}: \rho=0$ will be rejected in favor of an alternative hypothesis $\mathrm{H}_{0}: \rho>0$ if:

$$
-\ln \Lambda_{\rho=0}>\left(1+\frac{\hat{\delta}_{\rho=0}}{1+\hat{\delta}_{\rho=0}}\right) \chi_{\frac{\left(1+\hat{\delta}_{\rho=0}\right)^{2}}{\left(1+2 \hat{\delta}_{\rho=0}\right)} \mathrm{df}, \alpha}
$$

where, the right side is the critical value based on the $100(1-a)^{\text {th }}$ percentile of the central chi squared distribution with $\frac{\left(1+\hat{\delta}_{\rho=0}\right)^{2}}{\left(1+2 \hat{\delta}_{\rho=0}\right)}$ df and a significance level $\mathrm{a} \in$ $(0,1)$. We now write the p-value for rejecting the null hypothesis in favor of an alternative hypothesis and it is:

$$
\begin{aligned}
& \mathrm{p} \text { - value } \\
& =\operatorname{Pr}\left[\chi_{\frac{\left(1+\hat{\delta}_{\rho=0}\right)^{2}}{\left(1+2 \hat{\delta}_{\rho=0}\right)}}^{2} \mathrm{df}<\frac{-\ln \Lambda_{\rho=0}}{\left(1+\frac{\hat{\delta}_{\rho=0}}{1+\hat{\delta}_{\rho=0}}\right)}\right]
\end{aligned}
$$

The statistical power of the test statistic in Equation (29) can be calculated with a selection of a specific value for $\rho^{*}$ in the alternative hypothesis. The statistical power is the probability of rejecting the null hypothesis $\mathrm{H}_{0}: \rho=$ 0 in favor of an alternative hypothesis $\mathrm{H}_{1}: \rho=\rho^{*} \neq 0$. Under the alternative hypothesis, the minus log likelihood ratio: 


$$
\begin{aligned}
& -\ln \Lambda_{\rho^{*}}=\ln L(\hat{\rho}, \hat{\tau})-\ln \mathrm{L}\left(\rho=\rho^{*}, \hat{\tau}_{\rho=\rho^{*}}\right) \\
& =\mathrm{n}\left|\left(1-\frac{\bar{y}^{2}}{\mathrm{~s}^{2}}\right)\left\{1+\frac{1+\left(1-\frac{\bar{y}^{2}}{\mathrm{~s}^{2}}\right)^{2}}{1+\left(1-\frac{\bar{y}^{2}}{\mathrm{~s}^{2}}\right)}\right\}-2 \rho^{*} \bar{y}\right|
\end{aligned} \mid
$$

Follows a non-central chi-squared distribution with one df and non-centrality parameter:

$$
\hat{\delta}_{\rho^{*}}=\left(\hat{\rho}-\rho^{*}\right) / \operatorname{vâr}(\hat{\rho})=\left|\left(1-\frac{\rho^{*}}{\hat{\rho}}\right)\left(\frac{\bar{y}}{s^{2}}\right)^{3} / n\right|
$$

This non-central chi squared distribution with one df and non-centrality parameter $\hat{\delta}_{\rho}$ is approximately $\left(1+\frac{\hat{\delta}_{\rho^{*}}}{1+\hat{\delta}_{\rho^{*}}}\right)$ times a central chi squared score with $\frac{\left(1+\hat{\delta}_{\rho^{*}}\right)^{2}}{\left(1+2 \hat{\delta}_{\rho^{*}}\right)}$ df. The power is the probability of accepting a true alternative hypothesis $\mathrm{H}_{1}: \rho=\rho^{*}$. That is:

$$
\begin{aligned}
& \text { Power } \\
& =\operatorname{Pr}\left[\chi_{\frac{\left(1+\hat{\delta}_{\rho^{*}}^{2}\right)^{2}}{\left(1+2 \hat{\delta}_{p^{*}}\right)^{2}} \mathrm{df}}\right. \\
& \left.<\frac{\left(1+\frac{\hat{\delta}_{\rho=0}}{1+\hat{\delta}_{\rho=0}}\right)\left(-\ln \Lambda_{\rho^{*}}\right) \chi_{\frac{\left(1+\hat{\rho}_{\rho=0}\right)^{2}}{\left(1+2 \hat{\delta}_{\rho=0}\right)}}^{2} \mathrm{df}, \alpha}{\left(1+\frac{\hat{\delta}_{\rho^{*}}}{1+\hat{\delta}_{\rho^{*}}}\right)\left(-\ln \Lambda_{\rho=0}\right)}\right] .
\end{aligned}
$$

The previously mentioned results are now illustrated using bladder cancer recurrence times data in Andrews and Herzberg (1985). The patients within each group are compared in Table 2-5. The expected recurrence time based on prolonged exponential distribution in Equation (5a), the MLE of the medication's effect's and the pvalue are calculated using Equations (6a), (28) and (30). They are displayed in Table 1-5. The hypothesis $\mathrm{H}_{0}$ : $\rho=$ 0 is rejected when p-value is small. Using LRT statistic in Equation (31) and power formula in Equation (32), the power, the probability of accepting hypothesis $\mathrm{H}_{1}$ : $\rho^{*}=1$ is calculated and summarized in the Table 1-4. The power of LRT is excellent in all groups.

The patients in pyridoxine group are more homogeneous than those in other two groups. But, the expected first order statistic is smaller in placebo group than in other two groups and it suggests the importance of medical medication's effect to bladder cancer patients. The expected recurrence time for 2 nd order statistic adjusted for the 1st order statistic (Table 2) reveals that the thiotepa medication's effect delays it more. The correlation (Table 2) between record quicker and record longer bladder cancer recurrence time is indicative of no significant connection which is good.

The sketch of expected recurrence time in terms $\tau$ for an estimated $\hat{\rho}$ (Fig. 4a) and in terms of $\rho$ for an estimated $\hat{\tau}$ (Fig. 4b) based on prolonged exponential distribution in Equation (5a) reveals that the recurrence is prolonged because of an effective medication's effect (that is, as $\hat{\rho} \rightarrow \infty$ ). The prolonging is moderate when $\hat{\tau}$ increases. The exponential distribution in Equation (5b) has no capability to illustrate the medication's effect.

The survival probability without a recurrence at a given time is sketched for each group (Blue curve for placebo, green curve for thiotepa and pink curve for pyridoxine) in Fig. 4a using exponential distribution in Equation (5b) and in Fig. 4b. Using prolonged exponential distribution in Equation (5a). Notice that prolonged exponential distribution in Equation (5a) but not exponential distribution in Equation (5b) clearly distinguishes pyridoxine and thiotepa groups. This finding advocates the critical role of prolonging parameter $\rho$. Let us scrutinize below how patients perform compared to each other within a group.

\subsection{Patients in Placebo Group}

The patients in placebo group are now compared using exponential distribution in Equation (5b) and prolonged exponential distribution in Equation (5a). The expected mean recurrence time using exponential distribution in Equation (5b) over-estimated for 3rd, 5th, 6th and 7th patient and under-estimated for 4th patient, as this process does not take into account the impact of the medication's effect Fig. 4c-4g. However, in almost all patients (except the first and fourth case), the prolonged exponential distribution in Equation (5a) is better than exponential distribution in Equation (5b) according to their p-values (Table 4). In the first and fourth patient, the model exponential distribution in Equation (5b) and the model prolonged exponential distribution in Equation (5b) are the same as their $\hat{\rho}$ value is not small (Table 3). 
The power of accepting hypothesis $H_{1}: \rho^{*}=1$ is excellent for all patients except it is moderately high for the sixth patient. The survival probability based on prolonged exponential distribution in Equation (5a) in terms of survival probability based on exponential distribution in Equation (5b) is sketched for patients in Fig. 5a-5c. The difference in survival probabilities is negligible when the configuration shows no convexity. The Fig. 5a shows no convexity confirming that the medication's effect has no impact on 1st patient. All other patients in placebo group received medication' effect as their figures shows convexity.Now, patients in thiotepa group are compared using exponential distribution in Equation (5b) and prolonged exponential distribution in Equation (5a). The exponential distribution in Equation $(5 \mathrm{~b})$ over-estimated the expected recurrence time for 1 st and 2 nd patient as it does not show medication's effect. In 1st and 2nd patient, the prolonged exponential distribution in Equation (5a) fits better, according to their p-values (Table 5). In the third patient, the models exponential distribution in Equation (5b) and prolonged exponential distribution fit equally well as their $\hat{\rho}$ value is negligible.

The power of accepting hypothesis $\mathrm{H}_{1}: \rho^{*}=1$ is excellent for 2 nd and 3 rd but only moderately high for 1st patient (Table 4). Consequently, any assessment of their survival probability could be different depending on whether exponential distribution in Equation (5b) or prolonged exponential distribution in Equation (5a) is selected. Their difference is negligible only when the configuration has no convexity. But, only the Fig. 5c shows no convexity confirming that the medication's effect has no impact on 3rd patient.

Now, patients in the pyridoxine group are compared using exponential distribution in Equation (5b) and prolonged exponential distribution in Equation (5a). The exponential distribution in Equation (5b) over-estimated the expected recurrence time in all cases because it does not take into account the impact of drug in the medication's effect.

Table 2. Comparison of three groups based on prolonged exponential distribution (5a)

\begin{tabular}{lllr}
\hline Group & $\mathrm{E}\left[\mathrm{Y}_{(1)}\right]$ & $\mathrm{E}\left[\mathrm{Y}_{(2)} \mid \mathrm{Y}_{(1)}\right]$ & Corr (U,V) \\
\hline Placebo & 0.111 & 0.02 & 0.1791 \\
Thiotepa & 0.223 & 0.09 & 0.2887 \\
Pyridoxine & 0.042 & 0.01 & 0.1626 \\
\hline
\end{tabular}

Table 3. Summary of results for patients in placebo group

\begin{tabular}{lllllll}
\hline & $\begin{array}{c}\text { Expected recurrence } \\
\text { Patient } \\
\text { time with }(5 \mathrm{~b})\end{array}$ & $\begin{array}{c}\text { Expected recurrence } \\
\text { time with }(5 \mathrm{a})\end{array}$ & $\bar{\tau}$ in $(5 \mathrm{a})$ & $\bar{\rho}$ in $(5 \mathrm{a})$ & $\begin{array}{l}\mathrm{p} \text {-value to test } \\
\mathrm{H}_{0}: \rho=0\end{array}$ & $\begin{array}{l}\text { Power to accept } \\
\mathrm{H}_{1}: \rho=1\end{array}$ \\
\hline 1 & 5.20 & 5.200 & 4.750 & 0.0220 & 0.33 & 1.00 \\
2 & 6.67 & 6.667 & 3.340 & 74.7010 & 0.00 & 0.94 \\
3 & 6.00 & 2.548 & 1.583 & 0.9845 & $2 \mathrm{E}-08$ & 0.93 \\
4 & 10.60 & 15.850 & 13.710 & 0.0135 & 0.128 & 0.00 \\
5 & 5.78 & 3.321 & 1.981 & 1.0554 & $5.00 \mathrm{E}-09$ & 0.99 \\
6 & 8.20 & 2.629 & 1.671 & 0.8044 & $6 \mathrm{E}-15$ & 0.72 \\
7 & 6.13 & 6.125 & 4.405 & 0.1454 & 0.008 & 1.00 \\
\hline
\end{tabular}

Table 4. Summary of results in thiotepa Group (G2)

\begin{tabular}{|c|c|c|c|c|c|c|}
\hline Patient & $\begin{array}{l}\text { Expected recurrence } \\
\text { time with }(5 b)\end{array}$ & $\begin{array}{l}\text { Expected recurrence } \\
\text { time with }(5 a)\end{array}$ & $\bar{\tau}$ in $(5 a)$ & $\bar{\rho}$ in $(5 a)$ & $\begin{array}{l}\text { p-value to test } \\
\mathrm{H}_{0}: \rho=0\end{array}$ & $\begin{array}{l}\text { Power to accept } \\
\mathrm{H}_{1}: \rho=1\end{array}$ \\
\hline$\overline{1}$ & 2.00 & 0.385 & 0.250 & 4.6667 & $4 \mathrm{E}-21$ & 0.57 \\
\hline 2 & 5.29 & 4.391 & 2.378 & 2.3110 & $3 \mathrm{E}-10$ & 0.93 \\
\hline 3 & 7.60 & 7.60 & 6.092 & 0.0540 & 0.109 & 1.00 \\
\hline
\end{tabular}

Table 5. Summary of results in pyridoxine Group (G3)

\begin{tabular}{lllllll}
\hline & $\begin{array}{l}\text { Expected recurrence } \\
\text { time with }(5 \mathrm{~b})\end{array}$ & $\begin{array}{l}\text { Expected recurrence } \\
\text { time with }(5 \mathrm{a})\end{array}$ & $\bar{\tau}$ in $(5 \mathrm{a})$ & $\bar{\rho}$ in $(5 \mathrm{a})$ & $\begin{array}{l}\mathrm{p} \text {-value to test } \\
\mathrm{H}_{0}: \rho=0\end{array}$ & $\begin{array}{l}\text { Power to accept } \\
\mathrm{H}_{1}: \rho=1\end{array}$ \\
\hline 1 & 6.80 & 3.020 & 1.868 & 0.8617 & $8 \mathrm{E}-08$ & 0.98 \\
2 & 5.00 & 0.609 & 0.400 & 2.7381 & $5 \mathrm{E}-76$ & 1.00 \\
3 & 4.33 & 1.655 & 1.038 & 1.4061 & $1 \mathrm{E}-16$ & 0.31 \\
4 & 4.67 & 1.701 & 1.071 & 1.3296 & $1 \mathrm{E}-18$ & 0.04 \\
5 & 5.00 & 5.000 & 3.200 & 0.4018 & $5 \mathrm{E}-04$ & 1.00 \\
6 & 6.13 & 5.136 & 2.773 & 2.0854 & $4 \mathrm{E}-12$ & 0.95 \\
\hline
\end{tabular}


Ramalingam Shanmugam / American Medical Journal 4 (1): 43-62, 2013

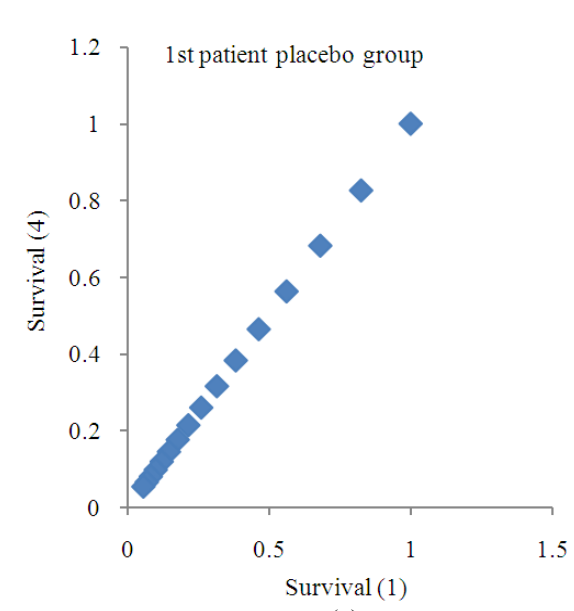

(a)

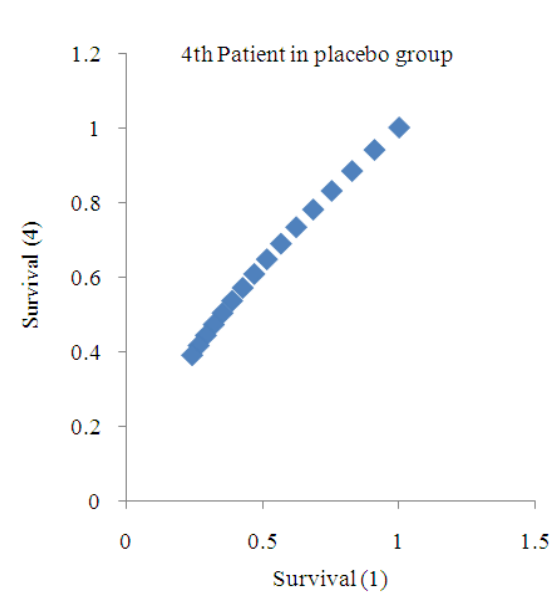

(d)

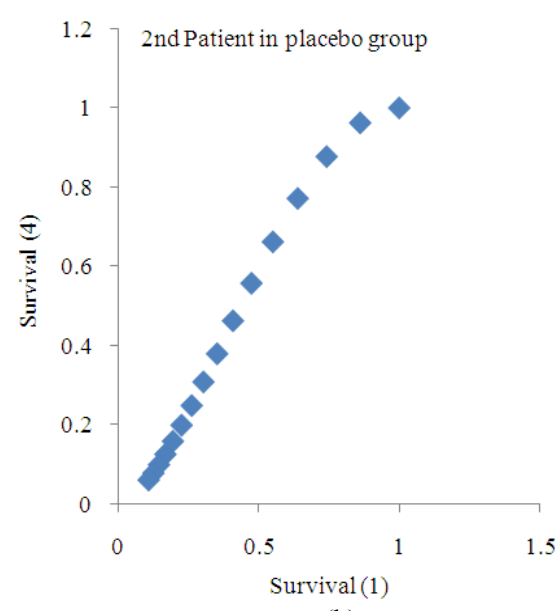

(b)

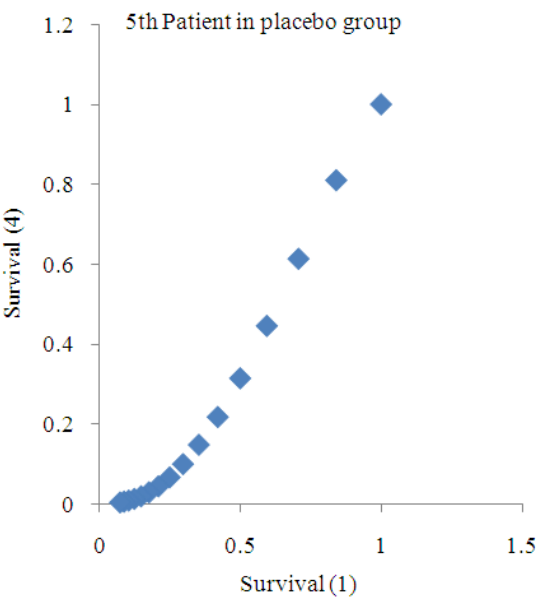

(e)

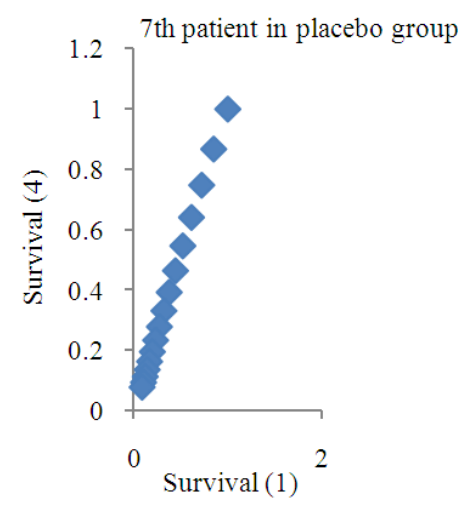

(g)

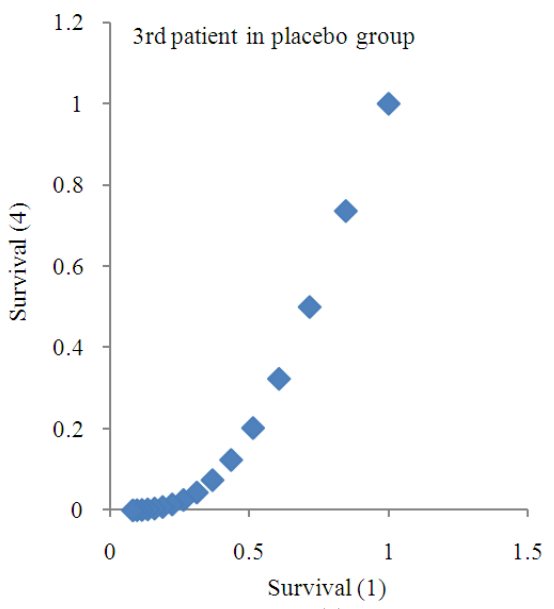

(c)

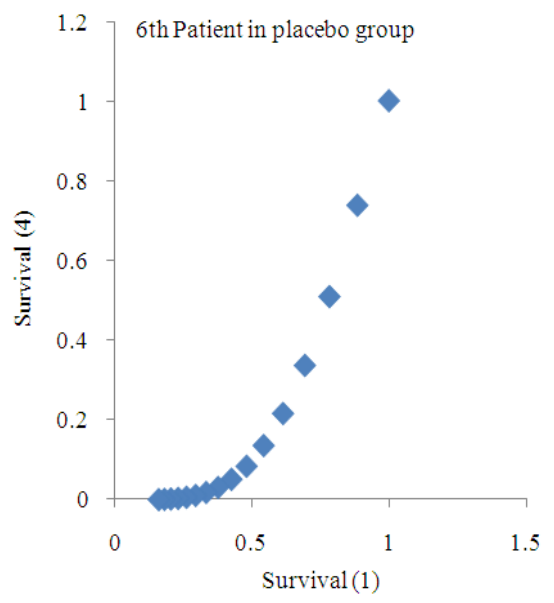

(f)

Fig. 4. (a) Survival of Patient 1 in placebo (b) Survival of Patient 2 in placebo (c) Survival of Patient 3 in placebo (d) Survival of Patient 4 in placebo (e) Survival of Patient 5 in placebo (f) Survival of Patient 6 in G1 (g) Survival of Patient 7 in placebo 
Ramalingam Shanmugam / American Medical Journal 4 (1): 43-62, 2013

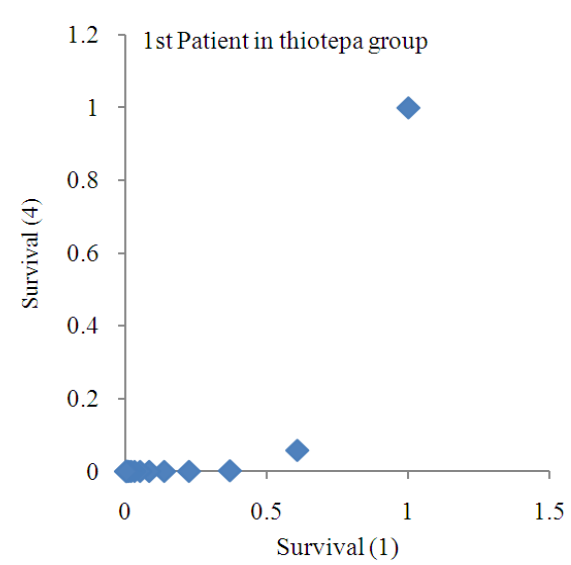

(a)

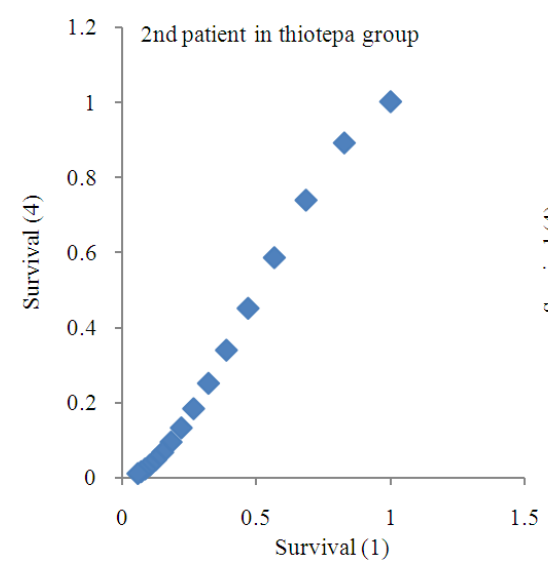

(b)

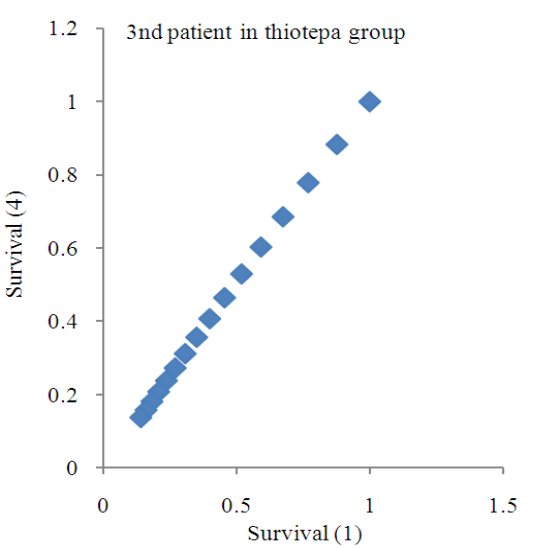

(c)

Fig. 5. (a) Survival of Patient 1 in thiotepa (b) Survival of Patient 2 in thiotepa (c) Survival of Patient 1 in thiotepa

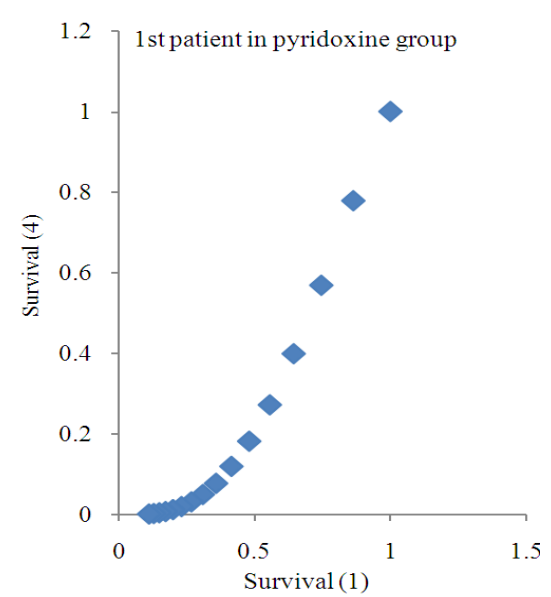

(a)

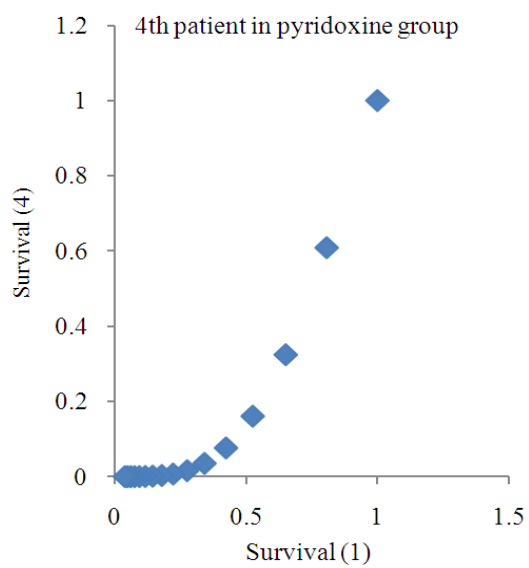

(d)

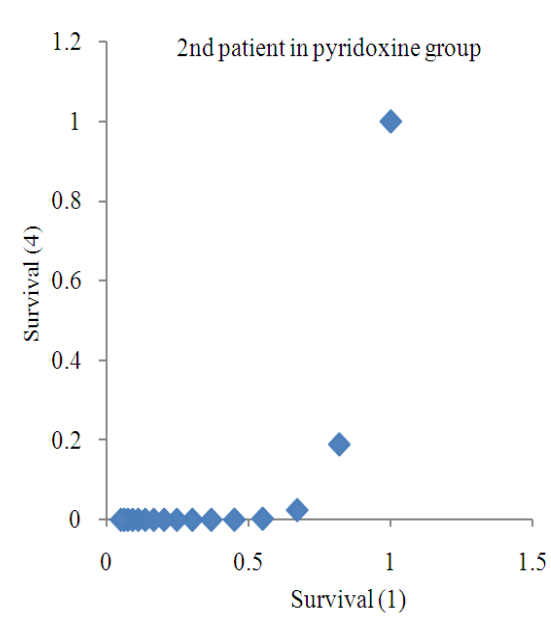

(b)

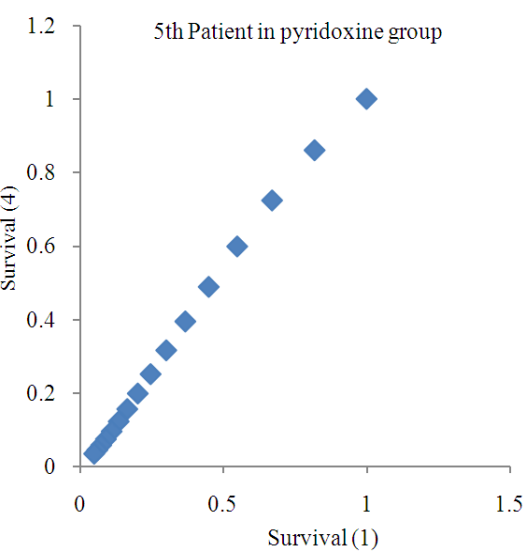

(e)

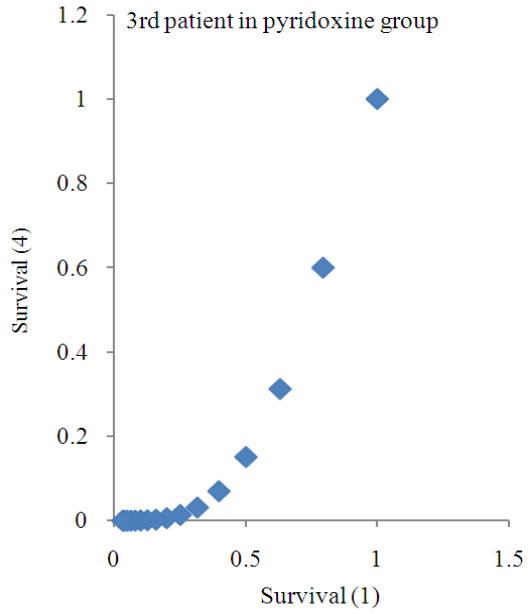

(c)

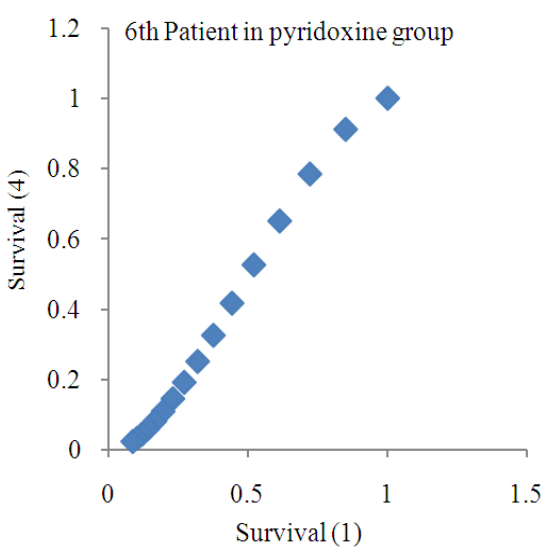

(f)

Fig. 6. (a) Survival of Patient 1 in pyridoxine (b) Survival of Patient 2 in pyridoxine (c) Survival of Patient 3 in pyridoxine (d) Survival of Patient 4 in pyridoxine (e) Survival of Patient 5 in pyridoxine (f) Survival of Patient 6 in pyridoxine 
The prolonged exponential distribution in Equation (5a) implies that the medication's effect has a significant impact in all patients, according to their p-values (Table 5). The power of accepting hypothesis $\mathrm{H}_{1}: \rho^{*}=1$ is excellent for 1st, 2nd, 5th and 6th, is moderate for 3rd, but is poor for 4th patient (Table 5). The survival probability based on prolonged exponential distribution in Equation (5a) in terms of survival probability based on exponential distribution in Equation (5b) is sketched for patients in Fig. 6a-6f. The difference in survival probabilities is negligible when the configuration shows no convexity.

\section{CONCLUSION}

The importance of configuring the impact of medication's effect is witnessed in all three groups. The prolonged exponential distribution in Equation (5a) is versatile, compared to the exponential distribution in Equation (5b), to capture and test the significance of the medication's effect to prolong the bladder cancer recurrence time. While not all patients exhibit same predicable recurrence time, their difference could be well explained using a regression methodology with covariates. The research work to develop regression methodology is underway to be reported in another article.

\section{REFERENCES}

Ahsanullah, M., 1978. Record values and its exponential distribution. Annals Instit. Stat. Math., 30: 429-433.

Ahsanullah, M., 1995. Record Statistics. 1st Edn., Nova Science Publishers, Commack, New York, ISBN10: 1560722037, pp: 224.
Andrews, D.F. and A.M. Herzberg, 1985. Data: A Collection of Problems from Many Fields for the Student and Research Worker. 1st Edn., Springer, New York, ISBN-10: 0387961259, pp: 442.

Beecher, H.K., 1955. The powerful placebo. J. Am. Med. Assoc., 159: 1602-1606. DOI: 10.1001/jama.1955.02960340022006

Blumenfeld, D., 2010. Operations Research Calculations Handbook. 2nd Edn., Taylor and Francis, Boca Raton, ISBN-10: 1420052411, pp: 256.

Kashanian, M., R. Mazinani and S. Jalalmanesh, 2007. Pyridoxine (vitamin B6) therapy for premenstrual syndrome. Int. J. Gynecol. Obstetrics, 96: 43-44. PMID: 17187801

Lindsey, K., 1997. Modelling Frequency and Count Data. 1st Edn., Oxford University, Oxford, UK.

Panjer, H., 2006. Operational Risk: Modeling and Analytics. 1st Edn., John Wileyand Sons, Hoboken, ISBN-10: 9780470051306, pp: 512.

Stuart, A. and K. Ord, 1994. Kendall's Advanced Theory of Statistics. 1st Edn., Griffin Publication, London, UK.

Wald, A., 1943. Tests of statistical hypotheses concerning several parameters when the number of observations is large. Trans. Am. Math. Soc., 54: 426-482. 